A partitioned correlation function interaction approach for describing electron correlation in atoms

This content has been downloaded from IOPscience. Please scroll down to see the full text. 2013 J. Phys. B: At. Mol. Opt. Phys. 46085003

(http://iopscience.iop.org/0953-4075/46/8/085003)

View the table of contents for this issue, or go to the journal homepage for more

Download details:

IP Address: 130.235.66.10

This content was downloaded on 05/11/2013 at 23:03

Please note that terms and conditions apply. 


\title{
A partitioned correlation function interaction approach for describing electron correlation in atoms
}

\author{
S Verdebout $^{1}$, P Rynkun ${ }^{2}$, P Jönsson ${ }^{3}$, G Gaigalas $^{4}$, C Froese Fischer ${ }^{5}$ \\ and M Godefroid ${ }^{1}$ \\ ${ }^{1}$ Chimie Quantique et Photophysique, CP160/09, Université Libre de Bruxelles, Av FD Roosevelt 50, \\ B-1050 Brussels, Belgium \\ ${ }^{2}$ Lithuanian University of Educational Sciences, Studentu 39, Vilnius, LT-08106, Lithuania \\ ${ }^{3}$ Group for Materials Science and Applied Mathematics, Malmö University, 20506 Malmö, Sweden \\ ${ }^{4}$ Vilnius University Research Institute of Theoretical Physics and Astronomy, A Goštauto 12, LT-01108 \\ Vilnius, Lithuania \\ ${ }^{5}$ Department of Computer Science, Vanderbilt University, Nashville, TN 37235, USA \\ E-mail: mrgodef@ulb.ac.be
}

Received 4 January 2013, in final form 28 February 2013

Published 4 April 2013

Online at stacks.iop.org/JPhysB/46/085003

\begin{abstract}
The traditional multiconfiguration Hartree-Fock (MCHF) and configuration interaction (CI) methods are based on a single orthonormal orbital basis. For atoms with many closed core shells, or complicated shell structures, a large orbital basis is needed to saturate the different electron correlation effects such as valence, core-valence and correlation within the core shells. The large orbital basis leads to massive configuration state function (CSF) expansions that are difficult to handle, even on large computer systems. We show that it is possible to relax the orthonormality restriction on the orbital basis and break down the originally very large calculations into a series of smaller calculations that can be run in parallel. Each calculation determines a partitioned correlation function (PCF) that accounts for a specific correlation effect. The PCFs are built on optimally localized orbital sets and are added to a zero-order multireference (MR) function to form a total wave function. The expansion coefficients of the PCFs are determined from a low dimensional generalized eigenvalue problem. The interaction and overlap matrices are computed using a biorthonormal transformation technique (Verdebout et al 2010 J. Phys. B: At. Mol. Phys. 43 074017). The new method, called partitioned correlation function interaction (PCFI), converges rapidly with respect to the orbital basis and gives total energies that are lower than the ones from ordinary MCHF and CI calculations. The PCFI method is also very flexible when it comes to targeting different electron correlation effects. Focusing our attention on neutral lithium, we show that by dedicating a PCF to the single excitations from the core, spin- and orbital-polarization effects can be captured very efficiently, leading to highly improved convergence patterns for hyperfine parameters compared with MCHF calculations based on a single orthogonal radial orbital basis. By collecting separately optimized PCFs to correct the MR function, the variational degrees of freedom in the relative mixing coefficients of the CSFs building the PCFs are inhibited. The constraints on the mixing coefficients lead to small off-sets in computed properties such as hyperfine structure, isotope shift and transition rates, with respect to the correct values. By (partially) deconstraining the mixing coefficients one converges to the correct limits and keeps the tremendous advantage of improved convergence rates that comes from the use of several orbital sets. Reducing ultimately each PCF to a single CSF with its own orbital basis leads to a non-orthogonal CI approach. Various perspectives of the new method are given.
\end{abstract}




\section{Introduction}

The electron correlation energy of an atom has been defined by Löwdin [1] as the difference between the exact nonrelativistic energy eigenvalue of the electronic Schrödinger equation and the energy of the single configuration state function (CSF) approximation, commonly called the Hartree-Fock (HF) energy. In line with this definition we think of electron correlation effects as those manifesting themselves beyond the HF approximation. It is useful to subdivide further and consider both static (nondynamical) and dynamical correlation [2]. Static correlation arises from near-degeneracies of the HF occupied and unoccupied orbitals. Systems with significant static correlation are poorly described by a single CSF and are said to have a strong multireference (MR) character. Dynamical correlation is due to the reduction in the repulsion energy related to the electron-electron cusp and is a shortrange effect.

Accurate description of electron correlation remains a major challenge in atomic structure calculations. To meet this challenge a number of different methods have been developed such as many-body perturbation theory (MBPT) [3,4], combinations of configuration interaction (CI) and many-body perturbation (CI+MBPT) [5-7], and coupled cluster [8-10] theories. Different kinds of variational methods have also been used, and one may specially note Hylleraas-type calculations, that explicitly include the interelectron distance $r_{12}$ in the construction of the wave function [11-14]. In quantum chemistry, variational complete active space self-consistent field (CASSCF) methods are quite successful for describing small and medium-size molecules, but are not sufficient when dynamical correlation must be included [15]. The latter are treated through second-order perturbation theory using a single or MR state as the zero-order approximation. Combined variational MR and second order Möller-Plesset perturbation calculations have also been applied very successfully by Ishikawa and co-workers $[16,17]$ to obtain accurate transition energies for a number of atomic systems. In this paper we will critically examine variational multiconfiguration methods, such as multiconfiguration Hartree-Fock (MCHF) combined with CI.

Multiconfiguration methods are quite general, and can be directly applied to excited and open-shell structures across the whole periodic table. By including the most important closely degenerate CSFs to form an MR expansion, the static correlation is efficiently captured. Dynamical correlation is accounted for by adding, to the MR expansion, CSFs obtained by single (S) and double (D) excitations from the CSFs in the MR set to an increasing set of active orbitals. The CSFs generated in this way build a space that we refer to as the correlation function (CF) space and it is convenient to think of multiconfiguration expansions as something built from CSFs in the MR space and in the CF space. Due to restrictions in the Racah or the Slater determinant algebra underlying the construction of the energy expressions, the orbitals are usually required to be orthonormal. Such an orthonormal orbital basis is not very efficient for larger systems. Let us consider an atomic system, for example $\mathrm{Ca}$, with several closed shells. To describe the dynamic correlation in the 1s shell we tailor an orbital set for which some of the orbitals should have a large overlap with the 1s radial orbital. Due to the orthogonality restrictions of the orbitals, the correlation in the $2 \mathrm{~s}$ shell needs to be described in terms of the previous radial orbitals, tailored for describing correlation within the 1s shell, as well as some new radial orbitals that are overlapping with the 2 s orbital etc. To capture the dynamic correlation between electrons in all the different shells, the orbital basis needs to be extended to a large number of orbitals for each symmetry, leading to massive CSF expansions [18]. This is in effect a scaling wall [19] that has been difficult to get around. In practice the electrons in the atom are considered as either core or outer valence electrons resulting in valence-valence, corevalence, and core-core types of SD excitations with the latter often neglected [18]. Another general problem with variational methods is that they are entirely based on the energy functional, and properties not strongly coupled to this functional may be inadequately described by the resulting wave function. As an example we consider the hyperfine interaction. The CSFs that are responsible for the important spin- and orbital-polarization effects are relatively unimportant for the total energy, and thus the orbital basis from the variational calculation may be spatially localized in such a way that the above effects are not captured. Alternatively, a very large orbital basis is needed to achieve convergence for these properties, leading to CSF expansions that grow unmanageably large.

The present work is an extension of a previous study of correlation energy in beryllium [20]. Based on a fast transformation technique, originally proposed by Malmqvist and collaborators [21,22], we show that it is possible to relax the orthonormality restriction on the orbital basis and use several mutually non-orthogonal orbital basis sets that are better adapted to the short range nature of the dynamical correlation. The gained freedom also makes it possible to tailor an orbital basis for capturing effects weakly connected to energy, improving convergence properties of atomic properties other than the energy. Partitioning the CF space into several subspaces, and using different orbital sets optimized for the different partitions, may be one way around the scaling wall associated with single orthonormal orbital sets.

\section{Partitioning the MCHF problem}

Starting from the non-relativistic Hamiltonian for an $\mathrm{N}$ electron system

$$
H=\sum_{i=1}^{N}\left[-\frac{1}{2} \nabla_{i}^{2}-\frac{Z}{r_{i}}\right]+\sum_{i<j}^{N} \frac{1}{r_{i j}},
$$

the MCHF approach determines an approximate wave function of the form

$$
\Psi\left(\gamma L S^{\pi}\right)=\sum_{i=1}^{M} c_{i} \Phi_{i}\left(\gamma_{i} L S^{\pi}\right),
$$

in which a CSF, $\Phi_{i}\left(\gamma_{i} L S^{\pi}\right)$, belongs either to the MR space or to the CF space. All CSFs have a given parity $(\pi)$ and $L S$ 
symmetry and they are built from a common basis of oneelectron spin-orbitals

$$
\phi\left(n l m_{l} m_{s}\right)=\frac{1}{r} P(n l ; r) Y_{l m_{l}}(\theta, \varphi) \chi_{m_{s}},
$$

where the radial functions $P(n l ; r)$ are to be determined [23]. For the approximate wave function (2), the integro-differential MCHF equations have the form

$$
\begin{gathered}
\left\{\frac{\mathrm{d}^{2}}{\mathrm{~d} r^{2}}+\frac{2}{r}[Z-Y(n l ; r)]-\frac{l(l+1)}{r^{2}}-\epsilon_{n l, n l}\right\} P(n l ; r) \\
=\frac{2}{r} X(n l ; r)+\sum_{n^{\prime} \neq n} \epsilon_{n l, n^{\prime} l} P\left(n^{\prime} l ; r\right)
\end{gathered}
$$

for the unknown radial functions [23]. The equations are coupled to each other through the direct $Y$ and exchange $X$ potentials and the Lagrange multipliers $\epsilon_{n l, n^{\prime} l}$. The Lagrange multipliers force the radial orbitals to be orthonormal within the same $l$ subspace. Under these conditions the CSFs are orthonormal

$$
\left\langle\Phi_{i} \mid \Phi_{j}\right\rangle=\delta_{i, j}
$$

The mixing coefficients appearing in the expansion over CSFs also enter in the form of the potentials and are determined by solving the CI problem

$$
\mathbf{H c}=E \mathbf{c},
$$

with $H_{i j}=\left\langle\Phi_{i}|H| \Phi_{j}\right\rangle$ being the Hamiltonian matrix and $\mathbf{c}=\left(c_{1}, c_{2}, \ldots, c_{M}\right)^{t}$ the column vector of mixing coefficients. For a given set of mixing coefficients, the equations (4) are solved by the self-consistent field (SCF) procedure. The SCF and CI problems are solved, one after the other, until convergence of both the radial functions and the selected CIeigenvector is achieved.

The strong coupling between the CSF expansion and the resulting optimized orbital basis is well known [24]. In the variational multiconfiguration approach indeed, the orbitals adapt spatially to account for the specific correlation effect targeted by the tailored expansion. In the present work, we investigate the possibility of breaking down the computational task into subtasks by partitioning the $\mathrm{CF}$ space into different subspaces, each targeting a specific correlation effect such as valence-correlation, core-valence or correlation within shells in the core, and performing separate MCHF calculations for each expansion built on the MR space and a CF subspace. In a final step the wave function is expanded in a basis consisting of CSFs from the MR space and functions built in each of the CF subspaces. The expansion coefficients are obtained by computing the Hamiltonian and overlap matrices and solving the corresponding generalized eigenvalue problem. The computation of the matrix elements between functions in the different subspaces, and this is the crucial point, is made possible by the biorthogonal transformation [21, 22].

The above scheme offers various advantages, resembling the 'divide and conquer' strategy-(i) from the computational point of view, smaller subtasks can be run in parallel, (ii) the resulting orbital basis sets are better adapted for capturing efficiently electron correlation, with the hope of getting a satisfactory accuracy for the desired property before reaching the scaling wall, (iii) the coupling between the subspaces reduces to a reasonably small dimension eigenvalue problem.

\section{The PCFI approach}

The efficiency of the method with a partitioned CF space was shown in our first paper [20], when targeting the total energy of the ground state of neutral beryllium. A MR expansion

$$
\left|\Psi^{\mathrm{MR}}\left(\gamma L S^{\pi}\right)\right\rangle=\sum_{j=1}^{m} a_{j}\left|\Phi_{j}^{\mathrm{MR}}\left(\gamma_{j} L S^{\pi}\right)\right\rangle,
$$

limited to the major contributions to valence correlation, including the near-degenerate $1 \mathrm{~s}^{2} 2 \mathrm{~s}^{2}$ and $1 \mathrm{~s}^{2} 2 \mathrm{p}^{2}$ configurations of the Layzer's complex, was corrected by three pair correlation functions $\left|\Lambda_{\mathrm{VV}}\right\rangle,\left|\Lambda_{\mathrm{CV}}\right\rangle,\left|\Lambda_{\mathrm{CC}}\right\rangle$ built by allowing single and double excitations from specific subshells of the MR CSFs to a given orbital active set (AS), and specifically tailored to describe the valence (VV), core-valence (CV) and core (CC) correlation effects. The final wave function

$$
\begin{aligned}
& \left|\Psi\left(\gamma L S^{\pi}\right)\right\rangle=\left|\Psi^{\mathrm{MR}}\left(\gamma L S^{\pi}\right)\right\rangle+\alpha_{\mathrm{VV}}\left|\Lambda_{\mathrm{VV}}\right\rangle+\alpha_{\mathrm{CV}}\left|\Lambda_{\mathrm{CV}}\right\rangle \\
& \quad+\alpha_{\mathrm{CC}}\left|\Lambda_{\mathrm{CC}}\right\rangle
\end{aligned}
$$

yielded a lower energy than the traditional MCHF method based on a very large CSF expansion.

In the present work, we generalize the approach by introducing the 'partitioned correlation functions' instead of 'pair correlation functions', preserving the PCF acronym, but allowing more flexible building rules for each PCF. For describing unambiguously the CSFs content of a given PCF, we first define a 'pure' PCF as a CSFs expansion containing only one kind of excitations, i.e. single (S), or double (D), or triple (T), . . excitations. We write such a PCF $\Lambda_{\{i\}}$, where the subscript $\{i\}$ specifies the set of occupied shells that are excited to a given AS. For instance, a PCF including only double excitations from the $n_{1} l_{1}$ and $n_{2} l_{2}$ subshells ${ }^{6}$ is written $\Lambda_{n_{1} l_{1} n_{2} l_{2}}$. A PCF could also be 'hybrid' if containing different kinds of excitations. Such a PCF may be seen as a superposition of 'pure' PCFs and is written as $\Lambda_{\{i\}-\{j\}-\ldots \text {, where for each family }}$ of excitations $\{i\},\{j\}, \ldots$, the letters sequence appearing in the subscript specify the $n l$-labels of the MR electrons that undergo the excitations. It is clear that a PCF in our approach does not fit with the usual definition of a pair correlation function $[25,26]$.

According to this notation, a PCF representing single excitations from $n_{1} l_{1}$ and double excitations from the $n_{1} l_{1}$ and $n_{2} l_{2}$ subshells, is written as

$$
\left|\Lambda_{n_{1} l_{1}-n_{1} l_{1} n_{2} l_{2}}\right\rangle=\sum_{n l} \alpha_{n l}\left|\Phi_{n_{1} l_{1}}^{n l}\right\rangle+\sum_{n l, n^{\prime} l^{\prime}} \alpha_{n l, n^{\prime} l^{\prime}}\left|\Phi_{n_{1} l_{1}, n_{2} l_{2}}^{n l, n^{\prime} l^{\prime}}\right\rangle
$$

where the first summation corresponds to all possible single excitations from the $n_{1} l_{1}$ shell for each CSF belonging to the MR set and the second one, to all possible double excitations from the $n_{1} l_{1}$ and $n_{2} l_{2}$ shells for each CSF belonging to the MR set. Defining $v$ as the $2 \mathrm{~s}$ or the $2 \mathrm{p}$ subshell $(v=\{2 \mathrm{~s}, 2 \mathrm{p}\})$, the beryllium ground state wave function (8) becomes

$$
\begin{aligned}
& \left|\Psi\left(\gamma L S^{\pi}\right)\right\rangle=\left|\Psi^{\mathrm{MR}}\left(\gamma L S^{\pi}\right)\right\rangle+\alpha_{\mathrm{VV}}\left|\Lambda_{v-v v}\right\rangle+\alpha_{\mathrm{CV}}\left|\Lambda_{1 \mathrm{~s} v}\right\rangle \\
& \quad+\alpha_{\mathrm{CC}}\left|\Lambda_{1 \mathrm{~s}-1 \mathrm{~s} 1 \mathrm{~s}}\right\rangle
\end{aligned}
$$

6 Note that $n_{1} l_{1}$ and $n_{2} l_{2}$ may refer to equivalent or non-equivalent electrons. 
where

- $\Lambda_{v-v v}$ is a hybrid PCF including S-excitations $(v)$ and D-excitations $(v v)$,

- $\Lambda_{1 \mathrm{~s} v}$ is a pure PCF including D-excitations involving one $1 \mathrm{~s}$ and one $v$ electrons,

- $\Lambda_{1 \mathrm{~s}-1 \mathrm{~s} 1 \mathrm{~s}}$ is a hybrid PCF including SD-excitations from the $1 \mathrm{~s}$ shell.

In order to avoid having the same CSF in two different PCFs, we consider the partitioning of the configuration space into disjoint sets. Another possible partition satisfying this property could be

$\left|\Psi\left(\gamma L S^{\pi}\right)\right\rangle=\left|\Psi^{\mathrm{MR}}\left(\gamma L S^{\pi}\right)\right\rangle+\alpha_{\mathrm{VV}}^{\prime}\left|\Lambda_{v-v v}\right\rangle+\alpha_{\mathrm{CV}}^{\prime}\left|\Lambda_{1 \mathrm{~s}-1 \mathrm{~s} v}\right\rangle$

$$
+\alpha_{\mathrm{CC}}^{\prime}\left|\Lambda_{1 \mathrm{~s} 1 \mathrm{~s}}\right\rangle
$$

where the single excitations $\left(1 \mathrm{~s} \rightarrow n_{1} l_{1}\right)$ have arbitrarily moved from the $\mathrm{CC}$ to the $\mathrm{CV}$ correlation function subspace. In the most general case, the MR function (7) is corrected by $p$ PCFs

$$
\left|\Psi\left(\gamma L S^{\pi}\right)\right\rangle=\left|\Psi^{\mathrm{MR}}\left(\gamma L S^{\pi}\right)\right\rangle+\sum_{i=1}^{p} \alpha_{i}\left|\Lambda_{i}\right\rangle,
$$

each of the PCFs corresponding to a given partition of the $\mathrm{CF}$ space:

$$
|\Lambda\rangle=\sum_{j}^{\operatorname{dim}(\Lambda)} c_{j}^{\Lambda}\left|\Phi_{j}^{\Lambda}\right\rangle .
$$

We use the notation $\Psi^{\Lambda}$ for the function consisting of the MR function and one of the correcting PCFs $\Lambda$ :

$$
\left|\Psi^{\Lambda}\left(\gamma L S^{\pi}\right)\right\rangle=\sum_{j=1}^{m} a_{j}^{\Lambda}\left|\Phi_{j}^{\mathrm{MR}}\left(\gamma_{j} L S^{\pi}\right)\right\rangle+\sum_{j}^{\operatorname{dim}(\Lambda)} c_{j}^{\Lambda}\left|\Phi_{j}^{\Lambda}\right\rangle .
$$

In our approach, this function is obtained by solving the corresponding MCHF equations (4)-(6) to optimize the $\Lambda$-PCF orbital set and mixing coefficients. Such a calculation that optimizes the MR eigenvector composition $\left\{a_{j}^{\Lambda}\right\}$ with orbitals frozen to the MR-MCHF solution (7), the mixing coefficients $\left\{c_{j}^{\Lambda}\right\}$ and the $\Lambda$-PCF radial functions, will be referred as a MR-PCF calculation. As far as the notation is concerned, we will underline when necessary the orbitals that are kept frozen during the SCF process. Solving the MCHF problem (12) for each $\Lambda_{i}(i=1,2, \ldots, p)$ produces $p$ mutually non-orthonormal one-electron orbital sets. Each of the orbital sets will be optimally localized for the correlation effect described by the corresponding PCF expansion. Assuming the CSFs of the MR and CF spaces orthonormal and $\left\langle\Psi^{\Lambda} \mid \Psi^{\Lambda}\right\rangle=1$, we have

$$
\left\langle\Psi^{\Lambda} \mid \Psi^{\Lambda}\right\rangle=\sum_{j=1}^{m}\left(a_{j}^{\Lambda}\right)^{2}+\sum_{j}^{\operatorname{dim}(\Lambda)}\left(c_{j}^{\Lambda}\right)^{2}=1,
$$

revealing that $\langle\Lambda \mid \Lambda\rangle \neq 1$. To keep a natural interpretation of the PCF weights, we renormalize each PCF according to

$$
|\bar{\Lambda}\rangle=\frac{1}{\sqrt{\sum_{j}\left(c_{j}^{\Lambda}\right)^{2}}}|\Lambda\rangle .
$$

The partitioned correlation function interaction (PCFI) approach consists in regrouping the $m$ components of the MR space and the $p$ CF subspaces in an a priori low-dimension interaction matrix to get a compact representation of the total wave function

$$
|\Psi\rangle=\sum_{i=1}^{m} a_{i}\left|\Phi_{i}^{\mathrm{MR}}\right\rangle+\sum_{j}^{p} b_{j}\left|\bar{\Lambda}_{j}\right\rangle,
$$

where the mixing coefficients $\left\{a_{i}\right\}$ and $\left\{b_{j}\right\}$ are obtained by solving the generalized eigenvalue problem ${ }^{7}$ of dimension $(M=m+p)$,

$$
\mathbf{H c}=E \mathbf{S c} .
$$

The corresponding Hamiltonian matrix may be explicitly written as

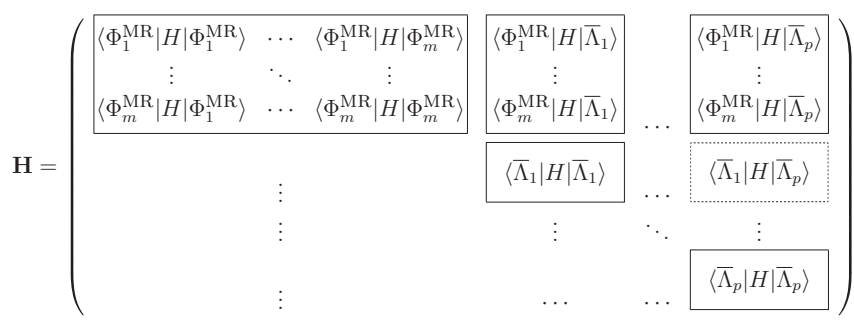

The matrix dimension $M$ is simply given by the sum of the number of CSFs belonging to the MR $(m)$ and the number of PCF functions $(p)$. The overlap matrix has the same structure, with a value of unity on the diagonal thanks to the renormalization $\left(\left|\Lambda_{i}\right\rangle \rightarrow\left|\bar{\Lambda}_{i}\right\rangle\right)$. It can be obtained by merely substituting the $H$ operator, appearing in each matrix element, by the unit operator. It reduces to the unit matrix for specific PCF-building rules as shown in appendix A. All plain-line blocks involve orthonormal orbitals and the construction of the matrix elements between the CSFs in the blocks is based on fast angular integration methods developed by Gaigalas et al $[27,28]$. This holds not only for the diagonal blocks, but also for blocks coupling the $\mathrm{CF}$ and the MR spaces since we do not allow the MR orbitals to vary in the MR-PCF MCHF calculations of (12). The building of all other blocks of the CF space, surrounded by a dashed line, involve non-orthogonal orbitals arising from independent MCHF calculations and requires therefore the use of biorthonormal transformations before the traditional methods for angular integration can be applied (see section 6 of [20]). By solving this compact eigenvalue problem, we showed in [20] that accurate total energies can be obtained.

However, as we already mentioned in that work, some variational freedom in the coefficients is lost by the fact that solving (14) does not allow relaxation in the relative weights within each PCF. The latter are indeed fixed linear combinations,

$$
|\bar{\Lambda}\rangle=\sum_{k} \bar{c}_{k}^{\Lambda}\left|\Phi_{k}\right\rangle
$$

and as such, we will refer to $|\bar{\Lambda}\rangle$ as a constrained CSFs expansion in the sense that the orbitals and the expansion

7 Note that the resulting $\left\{a_{i}\right\}$ and $\left\{b_{j}\right\}$ coefficients are such that $\langle\Psi \mid \Psi\rangle=1$ and that the $\left\{a_{i}\right\}$ coefficients differ from those of (7). 

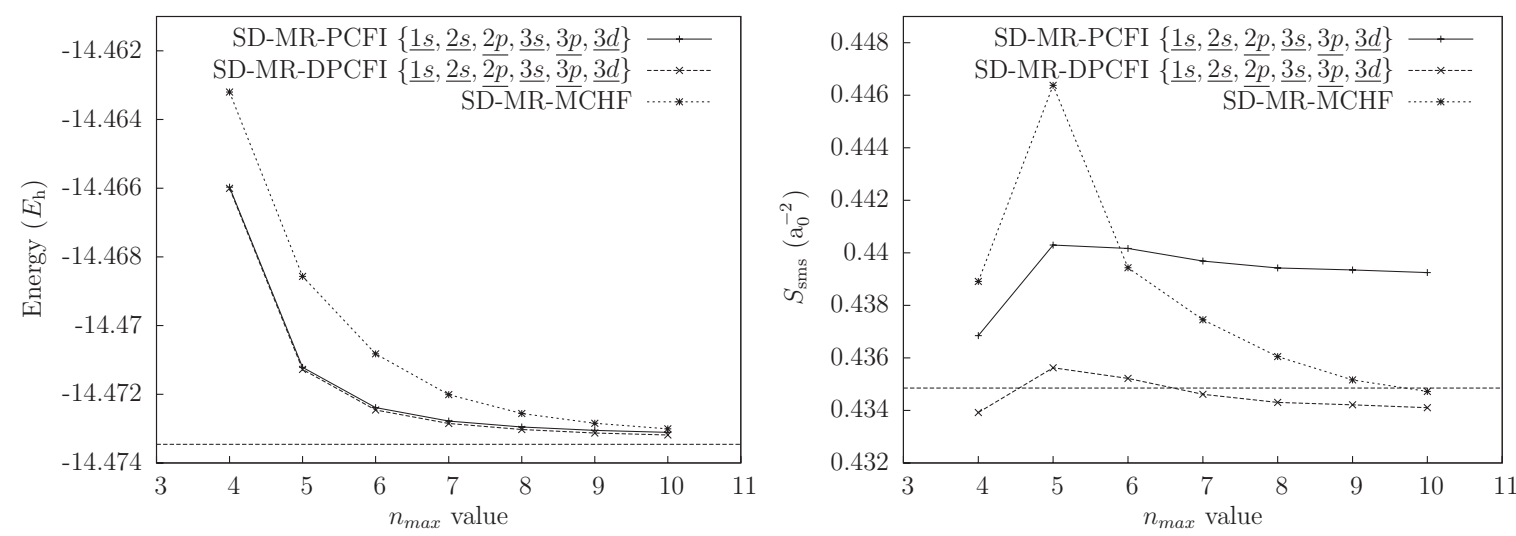

Figure 1. Be $1 \mathrm{~s}^{2} 2 \mathrm{~s} 2 \mathrm{p}^{1} \mathrm{P}^{\mathrm{o}}$. Comparison of the convergence trends between the SD-MR-PCFI, SD-MR-MCHF and SD-MR-DPCFI (see section 4) approaches for the total energy (left) and the SMS parameter (right). The $n_{\max }$ value specifies the orbital active set ( $n \leqslant n_{\max }$, without any angular limitation). The horizontal lines correspond to the results of Komasa and Rychlewski [29].

coefficients are not allowed to change. The coefficients $\left\{\bar{c}_{k}^{\Lambda}\right\}$ will be called the constrained coefficients. The effect associated with this loss of flexibility on a property, that we are investigating in the next section, will be qualified as the constraint effect.

\section{The constraint effect}

We were expecting that the constraint of keeping the $\left\{\bar{c}_{k}^{\Lambda}\right\}$ fixed to (16) would have a minor impact on all expectation values but extending our previous work [20] to $1 \mathrm{~s}^{2} 2 \mathrm{~s} 2 \mathrm{p}^{1} \mathrm{P}^{\mathrm{o}}$ of Be brought a surprise for spectroscopic properties other than the total energy. For this state, we optimized the following MR $(m=6)$ function

$$
\mathrm{MR}=1 \mathrm{~s}^{2}\left\{2 \mathrm{~s} 2 \mathrm{p}, 2 \mathrm{~s} 3 \mathrm{p}, 2 \mathrm{p} 3 \mathrm{~s}, 2 \mathrm{p} 3 \mathrm{~d}, 3 \mathrm{~s} 3 \mathrm{p}, 3 \mathrm{p} 3 \mathrm{~d}^{1} \mathrm{P}^{\mathrm{o}}\right\},
$$

that we corrected by three PCFs functions $(p=3)$ targeting respectively valence, core-valence and core correlation and built on single- and double-excitations (SD) from the MR set

$$
\begin{aligned}
& \left|\Psi\left(1 \mathrm{~s}^{2} 2 \mathrm{~s} 2 \mathrm{p}^{1} \mathrm{P}^{\mathrm{o}}\right)\right\rangle=\left|\Psi^{\mathrm{MR}}\left({ }^{1} \mathrm{P}^{\mathrm{o}}\right)\right\rangle+\alpha_{\mathrm{VV}}\left|\Lambda_{v-v v}\right\rangle \\
& \quad+\alpha_{\mathrm{CV}}\left|\Lambda_{1 \mathrm{~s} v}\right\rangle+\alpha_{\mathrm{CC}}\left|\Lambda_{1 \mathrm{~s}-1 \mathrm{~s} 1 \mathrm{~s}}\right\rangle
\end{aligned}
$$

where $v$ stands for any orbital of the valence MR set, i.e. $v=\{2 \mathrm{~s}, 2 \mathrm{p}, 3 \mathrm{~s}, 3 \mathrm{p}, 3 \mathrm{~d}\}$. After solving the MR-PCF problem (12) for the three $\Lambda \mathrm{s}$, we solved the $(M=m+p=9)$ eigenvalue PCFI problem. We will use the acronyms SDMR-PCFI and SD-MR-MCHF, respectively, for the present PCFI and for the conventional MR MCHF calculations. Figure 1 shows the striking difference in behaviour between the resulting energy and specific mass shift (SMS) parameter. While the total energies obtained with the SD-MR-PCFI and the SD-MR-MCHF models converge to the same limit, the two approaches give rise to an unexpected off-set on the SMS parameter.

For a deeper understanding of the ins and outs of the PCFI approach and fully appreciating its advantages, we moved to a smaller system: neutral lithium and its spectroscopic properties. For the lithium ground state, the HF approximation is rather good and the single reference $(m=1) 1 \mathrm{~s}^{2} 2 \mathrm{~s}^{2} \mathrm{~S}$ can be taken. We apply the PCFI method using two $(p=2)$ PCFs: (i) the first one targeting single and double excitations from the core (1s) orbital and denoted $\Lambda_{1 \mathrm{~s}-1 \mathrm{~s} 1 \mathrm{~s}}$, (ii) a second one, $\Lambda_{2 s-1 \mathrm{~s} 2 \mathrm{~s}}$, targeting single excitations from the $2 \mathrm{~s}$ valence shell and double excitations from the core (1s) and valence (2s) orbitals. The size of the PCFI matrix is small $(M=3)$. Figure 2 illustrates the MCHF radial functions of the $(n=5)$ AS resulting from the two MR-PCF equations applied to $\Lambda_{1 \mathrm{~s}-1 \mathrm{~s} 1 \mathrm{~s}}$ and $\Lambda_{2 s-1 \mathrm{~s} 2 \mathrm{~s}}$. As it was observed in [20, 24] for other systems, one can realize from this figure that a given PCF orbitals set specifically favours the region of the space occupied in the reference by the electrons undergoing excitations.

We compare the expectation values of other operators than the Hamiltonian, i.e. the SMS and the hyperfine interaction parameters [30], evaluated by the two SD-PCFI and SDMCHF methods in figure 3. The two curves illustrate the impact of the 'constraint effect' on three different properties: the total energy, the SMS $\left(S_{\text {sms }}\right)$ and the contact term $\left(a_{\text {cont }}\right)$. As mentioned in the end of section 3 , the origin of this effect is the hindrance to free variation in the expansion coefficients and in the orbitals. Even if the pre-optimized PCF orbital sets are fixed in the PCFI diagonalization step, the associated orbital constraint effect is expected to be small in comparison to the constraint on the mixing coefficients. The lack of variation in the orbitals is indeed somewhat compensated by the use of separated PCF orbital sets that makes the number of radial functions for a given AS, larger in the PCFI approach than in the MCHF method. Moreover the number of correlation layers used for a given PCF is probably large enough to reach saturation. The strongest limitation is likely to come from the fact that for each PCF, the mixing coefficients appearing in (16) are kept frozen in the interaction step that leads to the final many-electron wave function expression (13). By freezing these mixing coefficients, we inhibit the expression of any indirect effects. The relative weights of the CSFs within each PCF are indeed already fixed by the frozen coefficients $\left\{c_{j}^{\Lambda}\right\}$ obtained from each independent MR-PCF MCHF optimization of (12), each one targeting a specific correlation component, and no possibility is offered to these coefficients to capture the higher-order PCF-coupling effects.

Engels [31] studied the influence of various excitation classes on ab initio calculated isotropic hyperfine constants and showed how each class gives a direct contribution, 

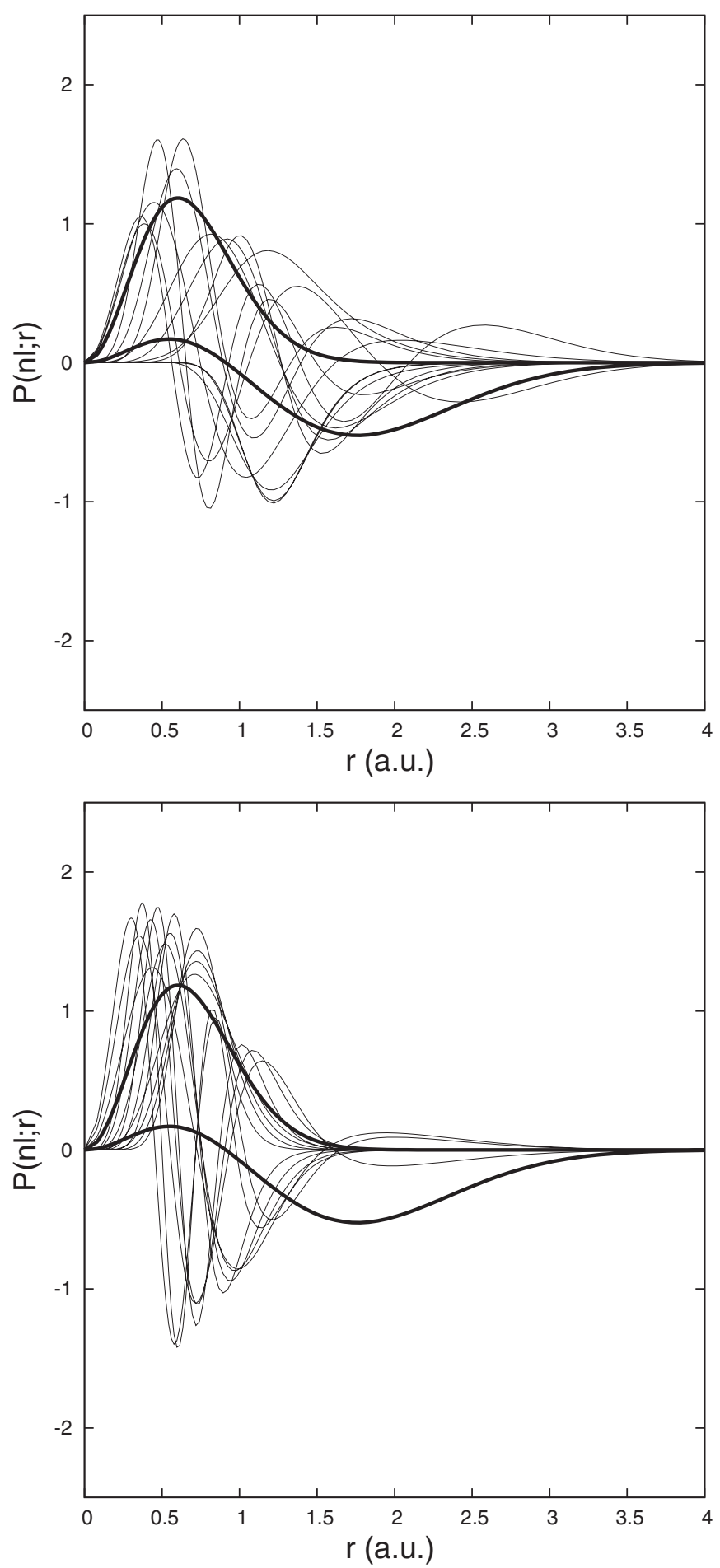

Figure 2. The two thick lines correspond to the spectroscopic Hartree-Fock $\underline{1 \mathrm{~s}}$ (no node) and $\underline{2 \mathrm{~s}}$ (one node) orbitals of $\mathrm{Li}$ $1 s^{2} 2 s^{2} S$. The other lines represent the radial functions of the correlation orbitals of the $n=5$ active set. The comparison between the top (core-valence MCHF) and bottom (core-core MCHF) figures illustrates the contraction of the first few correlation orbitals when going from $\Lambda_{2 s-1 s 2 s}$ to $\Lambda_{1 s-1 s 1 s}$ PCF calculations.

but that there are also important secondary effects on the contributions from the other classes due to relative changes of mixing coefficients. The importance of these indirect effects on the CSF weights, that affect computed properties quite substantially is confirmed in the present work. Back to the beryllium example, table 1 that collects the radiative data (length and velocity forms of the line strength $S_{l, v}$ and oscillator strength $g f_{l, v}$ as defined in equations (2)-(4) of [30]), for the resonance E1 transition of beryllium illustrates that the constraint effect can also be significant for properties involving more than one state. Comparing the $g f$ values from the SDMR-MCHF and SD-MR-PCFI calculations we see that the latter converges much more rapidly, but that there is an off-set of a little less than $1 \%$. Also, the agreement between the length and velocity forms is not as good for the PCFI method as for the ordinary MCHF.

\section{Deconstraining partitioned correlation functions}

In the PCFI method, the expansion coefficients for the CSFs in the PCF are constrained (locked) so that there is no possibility of relative changes due to the interaction with other PCFs. To recover this variational freedom, the PCFs can be deconstrained by transferring $h_{j}$ CSFs from the $j$ th PCF to the basis and at the same time setting their weights to zero, i.e. extending the PCFI space to

$$
\begin{aligned}
& \left\{\Phi_{1}^{\mathrm{MR}}, \ldots, \Phi_{m}^{\mathrm{MR}}\right\} \bigcup_{j=1}^{p}\left\{\bar{\Lambda}_{j}\right\} \\
& \quad \longrightarrow\left\{\Phi_{1}^{\mathrm{MR}}, \ldots, \Phi_{m}^{\mathrm{MR}}\right\} \bigcup_{j=1}^{p}\left\{\Phi_{1}^{j}, \ldots, \Phi_{h_{j}}^{j}, \bar{\Lambda}_{j}^{d}\right\} .
\end{aligned}
$$

The superscript $d$ for the PCF $\bar{\Lambda}_{j}^{d}$ indicates a renormalized $d e$-constrained partitioned correlation function $(\mathrm{dPCF})$ whose weights of the transferred CSFs have been set to zero. The many-electron wave function expansion becomes

$$
|\Psi\rangle=\sum_{i=1}^{m} a_{i}\left|\Phi_{i}^{\mathrm{MR}}\right\rangle+\sum_{j=1}^{p}\left\{\sum_{k=1}^{h_{j}} c_{k}^{j}\left|\Phi_{k}^{j}\right\rangle+c_{j}\left|\bar{\Lambda}_{j}^{d}\right\rangle\right\},
$$

where the expansion coefficients are obtained from a higher dimension $\left(M=m+\sum_{j=1}^{p}\left(h_{j}+1\right)\right)$ a priori generalized eigenvalue problem. The size of each block in (15) involving at least one PCF is growing accordingly to the number of deconstrained CSFs. In the limit of the completely deconstrained case (i.e. $h_{j}=\operatorname{dim}\left(\bar{\Lambda}_{j}\right) \quad \forall j$ ), we regain full variational freedom in the coefficients, with the advantage that each CSF brings its tailored orbital basis. $M$ is then at his maximum value, i.e. the total number of CSFs, and the wave function (18) will be referred as being 'deconstrained'. It is strictly equivalent to a CI problem in the CSF space built on various mutually non-orthonormal orbital sets.

For solving the eigenvalue problem (14) and for building efficiently the interaction matrices associated with the selected operators in the basis of CSFs and dPCFs spanning the wave function (18), we modify the original way of presenting the biorthonormal transformation [22] to evaluate $O_{i j}=$ $\left\langle\Lambda_{i}|O| \Lambda_{j}\right\rangle$, where $O$ is the Hamilton or unit operator. Following the original formalism, we perform a biorthonormal transformation

$$
\left\langle\phi_{k}^{i} \mid \phi_{m}^{j}\right\rangle=S_{k m}^{i j} \rightarrow\left\langle\tilde{\phi}_{k}^{i} \mid \tilde{\phi}_{m}^{j}\right\rangle=\delta_{n_{k}, n_{m}} \delta_{l_{k}, l_{m}}
$$



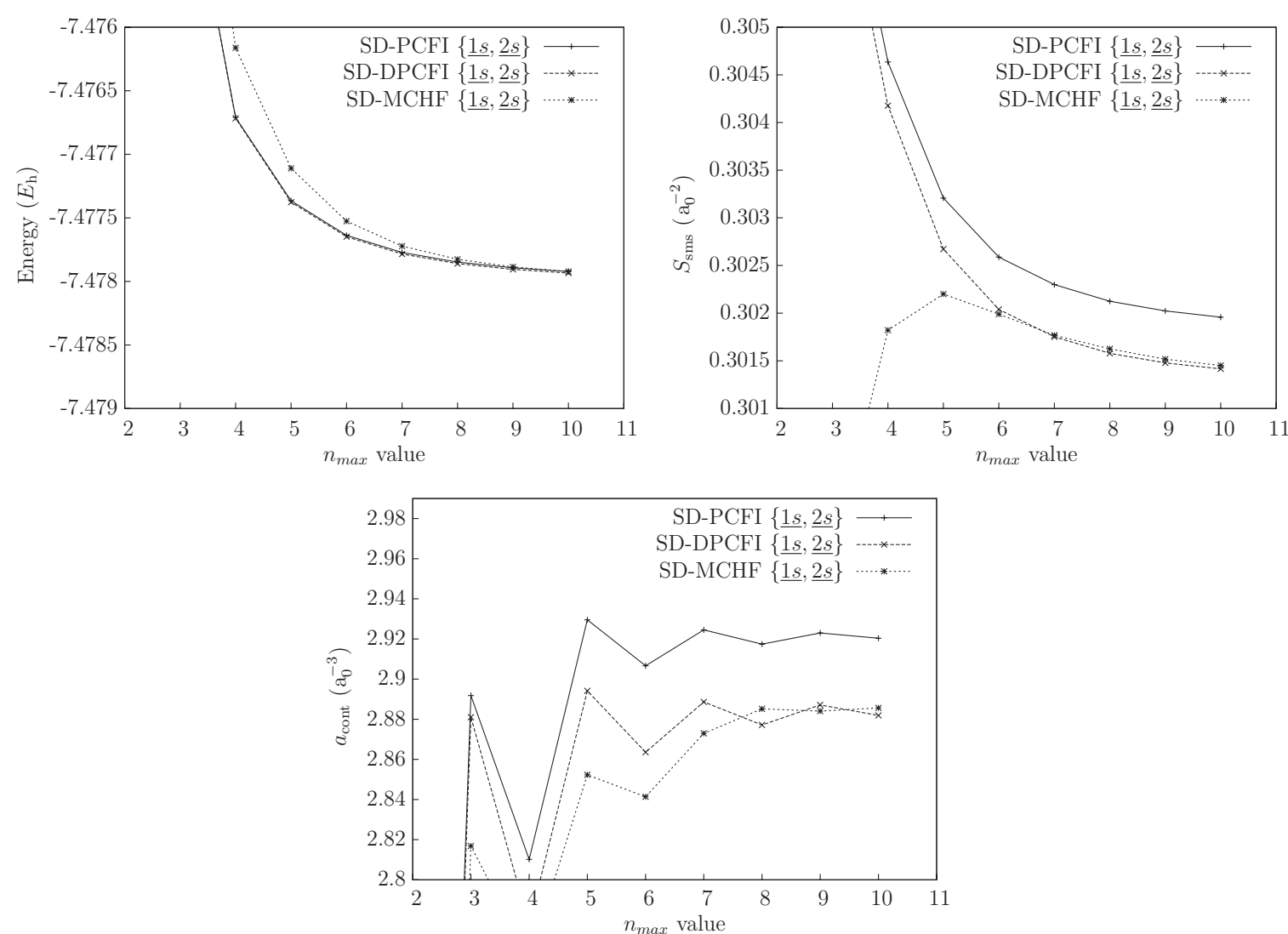

Figure 3. Convergence of the absolute total energy, the specific mass shift and the hyperfine contact parameters for the ground state of neutral lithium. The $n_{\max }$ value specifies the orbital active set $\left(n \leqslant n_{\max }\right.$, without any angular limitation). The agreement found between the SD-MCHF and SD-PCFI curves for the total energy is not observed for the two other properties. We recover the consistency with the traditional method by deconstraining the wave function (SD-DPCFI).

to express the original left- and right-hand side PCFs in the new CSF bases $\left\{\tilde{\Phi}_{k}^{i}\right\}$ and $\left\{\tilde{\Phi}_{l}^{j}\right\}$ :

$$
\begin{gathered}
\left|\Lambda_{i}\right\rangle=\sum_{k=1}^{n_{i}} d_{k}^{i}\left|\Phi_{k}^{i}\right\rangle=\sum_{k=1}^{n_{i}} \tilde{d}_{k}^{i}\left|\tilde{\Phi}_{k}^{i}\right\rangle \\
\left|\Lambda_{j}\right\rangle=\sum_{l=1}^{n_{j}} d_{l}^{j}\left|\Phi_{l}^{j}\right\rangle=\sum_{l=1}^{n_{j}} \tilde{d}_{l}^{j}\left|\tilde{\Phi}_{l}^{j}\right\rangle,
\end{gathered}
$$

where the counter-transformed eigenvectors $\left\{\tilde{d}_{k}^{i}\right\}$ and $\left\{\tilde{d}_{l}^{j}\right\}$ ensure the invariance of the total wave functions. Given the matrix representation $\tilde{\mathbf{O}}$ of an operator $O$

$$
\tilde{O}_{k l}=\left\langle\tilde{\Phi}_{k}^{i}|O| \tilde{\Phi}_{l}^{j}\right\rangle,
$$

the matrix element between these PCFs is written as

$$
\left\langle\Lambda_{i}|O| \Lambda_{j}\right\rangle=\sum_{k, l}\left(\tilde{d}_{k}^{i}\right)^{*} \tilde{d}_{l}^{j}\left\langle\tilde{\Phi}_{k}^{i}|O| \tilde{\Phi}_{l}^{j}\right\rangle=\left(\tilde{\mathbf{d}}^{i}\right)^{t} \tilde{\mathbf{O}}^{j}
$$

where $\tilde{\mathbf{d}}$ is the column vector of counter-transformed mixing coefficients. Note that each CSF expansion should be closed under de-excitation (CUD) for allowing the biorthonormal transformation [22, 20]. By strictly following this methodology, we may think that we should apply one biorthonormal transformation for each matrix element associated with any off-diagonal sub-matrix block involving two non-orthogonal orbital sets. However, as the countertransformation process is fixed by the overlap between the original spin-orbital bases, i.e. $\left\langle\phi_{k}^{i} \mid \phi_{m}^{j}\right\rangle=S_{k m}^{i j}$, we show in the appendix $B$ that one can evaluate the whole sub-matrix block by performing a single biorthonormal transformation treating simultaneously the counter-transformation of all the elements constituting the block-basis.

Using this strategy, relaxing the PCFI constraint to any degree becomes possible. The price to pay is the increase of the size of the PCFI problem (14). In the limit of the completely deconstrained case (i.e. $h_{j}=\operatorname{dim}\left(\bar{\Lambda}_{j}\right) \quad \forall j$ ), the PCFI approach is strictly equivalent to a CI problem in the original complete CSF space. We will use the label deconstrained partitioned correlation function interaction (DPCFI) for this 'deconstrained' approach. Since each CSF could be built, if worthwhile, on its own orbital basis, without any radial orthogonality constraints with the other ones, this DPCFI approach is equivalent to a general CI problem in nonorthogonal orbitals.

The (D)PCFI procedure can be summarized as follows.

(i) Perform a HF/MCHF calculation for the single/ multireference wave function (7).

(ii) Freeze the orbitals belonging to this MR space and perform $p$ separate MR-PCF MCHF calculations (12) for the different PCFs,

(iii) (optional) deconstrain each PCF by transferring the desired CSFs from the CF to the MR basis (see (17)), 
Table 1. Line strength $\left(S_{l}, S_{v}\right)$ and oscillator strength $\left(g f_{l}, g f_{v}\right)$ of the ${ }^{1} \mathrm{P}^{\mathrm{o}}-{ }^{1} \mathrm{~S}$ resonant line for the beryllium atom.

\begin{tabular}{|c|c|c|c|c|c|}
\hline \multicolumn{6}{|c|}{ SD-MR-MCHF } \\
\hline$n_{\max }$ & $\Delta E\left(\mathrm{~cm}^{-1}\right)$ & $S_{l}\left(\mathrm{e}^{2} \mathrm{a}_{0}^{2}\right)$ & $S_{v}\left(\mathrm{e}^{2} \mathrm{a}_{0}^{2}\right)$ & $g f_{l}$ & $g f_{v}$ \\
\hline 4 & 43513.26 & 10.6832 & 10.9355 & 1.41204 & 1.44539 \\
\hline 5 & 43069.99 & 10.6269 & 10.7335 & 1.39029 & 1.40424 \\
\hline 6 & 42843.85 & 10.6060 & 10.6049 & 1.38027 & 1.38013 \\
\hline 7 & 42686.81 & 10.6110 & 10.6255 & 1.37587 & 1.37774 \\
\hline 8 & 42635.54 & 10.6205 & 10.6301 & 1.37544 & 1.37668 \\
\hline 9 & 42606.57 & 10.6249 & 10.6366 & 1.37508 & 1.37659 \\
\hline 10 & 42593.62 & 10.6273 & 10.6375 & 1.37497 & 1.37628 \\
\hline \multicolumn{6}{|c|}{ SD-MR-PCFI $\{\underline{1 s}, \underline{2 s}, \underline{2 p}, \underline{3 s}, \underline{3 p}, \underline{3 d}\}$} \\
\hline$n_{\max }$ & $\Delta E\left(\mathrm{~cm}^{-1}\right)$ & $S_{l}\left(\mathrm{e}^{2} \mathrm{a}_{0}^{2}\right)$ & $S_{v}\left(\mathrm{e}^{2} \mathrm{a}_{0}^{2}\right)$ & $g f_{l}$ & $g f_{v}$ \\
\hline 4 & 42731.10 & 10.6848 & 10.6446 & 1.38686 & 1.38165 \\
\hline 5 & 42649.36 & 10.7012 & 10.7249 & 1.38634 & 1.38940 \\
\hline 6 & 42618.07 & 10.7047 & 10.7347 & 1.38578 & 1.38966 \\
\hline 7 & 42603.40 & 10.7083 & 10.7391 & 1.38576 & 1.38975 \\
\hline 8 & 42596.17 & 10.7096 & 10.7412 & 1.38570 & 1.38979 \\
\hline 9 & 42591.03 & 10.7104 & 10.7433 & 1.38563 & 1.38988 \\
\hline 10 & 42588.21 & 10.7110 & 10.7452 & 1.38561 & 1.39004 \\
\hline \multicolumn{6}{|c|}{ SD-MR-DPCFI $\{\underline{1 \mathrm{~s}}, \underline{2 \mathrm{~s}}, \underline{2 \mathrm{p}}, \underline{3 \mathrm{~s}}, \underline{3 \mathrm{p}}, \underline{3 \mathrm{~d}}\}$} \\
\hline$n_{\max }$ & $\Delta E\left(\mathrm{~cm}^{-1}\right)$ & $S_{l}\left(\mathrm{e}^{2} \mathrm{a}_{0}^{2}\right)$ & $S_{v}\left(\mathrm{e}^{2} \mathrm{a}_{0}^{2}\right)$ & $g f_{l}$ & $g f_{v}$ \\
\hline 4 & 42725.56 & 10.6443 & 10.5604 & 1.38143 & 1.37055 \\
\hline 5 & 42640.89 & 10.6355 & 10.6149 & 1.37755 & 1.37489 \\
\hline 6 & 42608.81 & 10.6324 & 10.6235 & 1.37612 & 1.37497 \\
\hline 7 & 42593.58 & 10.6358 & 10.6263 & 1.37606 & 1.37483 \\
\hline 8 & 42586.00 & 10.6361 & 10.6287 & 1.37586 & 1.37491 \\
\hline 9 & 42580.53 & 10.6363 & 10.6301 & 1.37570 & 1.37490 \\
\hline 10 & 42577.57 & 10.6369 & 10.6322 & 1.37569 & 1.37508 \\
\hline $\operatorname{CAS}\left(n_{\max } l_{\max }=9 \ell\right)$ & 42588.71 & 10.6234 & 10.6333 & 1.37431 & 1.37559 \\
\hline [32] & 42559.20 & & & 1.374 & \\
\hline [33] & 42593.44 & & & 1.3745 & 1.3759 \\
\hline [29] & 42560.52 & & & & \\
\hline [34] & 42670.69 & & & 1.340 & \\
\hline [35] & 42565.35 & & & & \\
\hline [36] & & & & $1.40 \pm 0.04$ & \\
\hline [37] & & & & $1.34 \pm 0.03$ & \\
\hline
\end{tabular}

(iv) build the Hamiltonian and other relevant operators interaction matrices (15) by performing the biorthonormal transformations, if necessary, using the weight matrix formalism (see appendix B),

(v) solve the eigenvalue problem (14) for getting the manyelectron wave function (13),

(vi) compute the desired property with the PCFI eigenvector(s).

As it is shown in appendix A, step (ii) allows to replace the overlap matrix equal of (14) by the unit matrix.

\section{Applications of the DPCFI method}

\subsection{Solving the constraint problem}

This DPCFI strategy has been applied to both lithium and beryllium by solving the eigenvalue problem in the original CSF basis instead of the PCF one. Each CSF comes with the orbital basis associated with the PCF from which it comes from. In both cases, as illustrated by figure 1 and figure 3 for beryllium and lithium respectively, the DPCFI values converge to the MCHF results, recovering all the indirect effects and keeping the tremendous advantage of a much faster convergence. This corroborates the fact that the problematic PCFI-MCHF discrepancy observed for the $S_{\text {sms }}$ and contact hyperfine parameters is due to the constraint effect. On the other hand, total energies are only slightly improved in the (PCFI $\rightarrow$ DPCFI) transition. A close analysis of the results displayed in figure 3 shows that for $n=10$, the relative differences (in absolute value) between the PCFI and DPCFI results are respectively $1.65 \times 10^{-4} \%, 0.18 \%$ and $1.33 \%$ for the total energy, the SMS and the contact term parameters. These numbers demonstrate that, compared with the total energy, the SMS and the contact term are much more sensitive to the constraint effect.

\subsection{The beryllium resonance line}

For the beryllium resonance transition (see table 1), passing from SD-MR-PCFI to SD-MR-DPCFI, the line strength is modified by about $0.7 \%$ in the length formalism and $1.1 \%$ in the velocity formalism, bringing the two forms in much closer 
agreement. The SD-MR-DPCFI is now in excellent agreement with the most extensive MCHF calculations available $(n=$ 9 CAS). As the absolute energy of each level, the transition energy is much less sensitive to the constraint effect since it undergoes a modification of less than $0.02 \%$. Note that both the SD-MR-PCFI and the SD-MR-DPCFI methods provide a transition energy at the $n=6$ level which is comparable to the SD-MR-MCHF $n=10$ result. As already pointed out in our first paper [20], the richness in terms of radial functions of the many-electron wave functions adopting different PCF definitely leads to a higher rate of convergence for all properties.

It is probably worthwhile to review briefly the status of the available oscillator strength values for this Be I resonance line reported in table 1. Experimentally, the situation has evolved since the publication in 1996 of the theoretical results of Fleming et al [33] of $g f_{l}=1.3745$ in the length form and $g f_{v}=1.3759$ in the velocity form. Irving et al [36] revised the old beam-foil measurement of Martinson et al [38], by including the cascade corrections through the ANDC analysis that increased the oscillator strength from $g f=$ $1.34 \pm 0.05 \rightarrow 1.40 \pm 0.04$, in nice agreement with the theoretical predictions. An independent measurement has been realized by Schnabel and Kock [37] using the cascade-free laser induced fluorescence method, yielding the original beamfoil result but with a smaller uncertainty, namely $1.34 \pm 0.03$. Amazingly, this value escaped to the attention of the authors of a rather complete compilation [39]. Table 1 shows that the present SD-MR-DPCFI results confirm the previous theoretical value [33], with a difference between the two gauges that is much smaller than the accuracy rating ' $A$ ' attributed by Fuhr and Wiese [40] to all transitions where this difference is smaller than $1.5 \%$. The small overlap between the two most recent experimental values [36, 37] calls for further investigations on the experimental side.

\subsection{The CAS-DPCFI approach in lithium}

In this section we present results obtained for selected spectroscopic properties involving the two lowest states of neutral lithium, i.e. $1 \mathrm{~s}^{2} 2 \mathrm{~s}^{2} \mathrm{~S}$ and $1 \mathrm{~s}^{2} 2 \mathrm{p}^{2} \mathrm{P}^{\mathrm{o}}$. The Li I ground state (D)PCFI calculations that were presented in section 4 and figure 3 to investigate the constraint effect are limited in the sense that triple excitations are systematically omitted in a SD calculation using a single configuration for the reference set. In order to obtain more accurate many-electron wave functions, we adopted the CAS list of CSFs combined with the DPCFI approach that remains manageable for a threeelectron system. For illustrating the great flexibility of the DPCFI approach, we investigate two different strategies-(i) treating core excitations globally and (ii) separating the single and double core-excitations and dedicating a PCF to corepolarization $(\mathrm{CP})$.

6.3.1. A global core description. Like in our first model, we use the HF solution as the zeroth-order wave function for the two lowest states of this three electron system. In this approach, we split the CF space made of single, double and triple excitations (SDT) in three different subspaces $(p=3)$ defining the following three PCFs:

- one for taking care of the inner-shell correlation between the two 1s electrons

$$
\begin{aligned}
& \left|\Lambda_{1 \mathrm{~s}-1 \mathrm{~s} 1 \mathrm{~s}}\right\rangle=\left|1 \mathrm{~s}^{2} 2 l^{2} L^{\pi}\right\rangle+\sum_{n^{\prime} l^{\prime}}\left|1 \mathrm{~s} 2 l n^{\prime} l^{\prime 2} L^{\pi}\right\rangle \\
& \quad+\sum_{n^{\prime} l^{\prime}, n^{\prime \prime} l^{\prime \prime}}\left|2 l n^{\prime} l^{\prime} n^{\prime \prime} l^{\prime \prime 2} L^{\pi}\right\rangle,
\end{aligned}
$$

where the summations run over the quantum numbers of the virtual (correlation) orbitals included in the considered $\mathrm{AS}$,

- a second one associated to the inter-shell correlation between the $1 \mathrm{~s}$ and the $2 l(l=\mathrm{s}$ or $\mathrm{p})$

$$
\begin{aligned}
& \left|\Lambda_{2 l-1 \mathrm{~s} 2 l}\right\rangle=\left|1 \mathrm{~s}^{2} 2 l^{2} L^{\pi}\right\rangle+\sum_{n^{\prime} l^{\prime}}\left|1 \mathrm{~s}^{2} n^{\prime} l^{\prime 2} L^{\pi}\right\rangle \\
& +\sum_{n^{\prime} l^{\prime}, n^{\prime \prime} l^{\prime \prime}}\left|1 \mathrm{~s} n^{\prime} l^{\prime} n^{\prime \prime} l^{\prime \prime} L^{\pi}\right\rangle,
\end{aligned}
$$

- a third and last one including the pure triple excitations

$$
\left|\Lambda_{1 \mathrm{~s} 1 \mathrm{~s} 2 l}\right\rangle=\left|1 \mathrm{~s}^{2} 2 l^{2} L^{\pi}\right\rangle+\sum_{n^{\prime} l^{\prime}, n^{\prime \prime} l^{\prime \prime}, n^{\prime \prime \prime} l^{\prime \prime \prime}}\left|n^{\prime} l^{\prime} n^{\prime \prime} l^{\prime \prime} n^{\prime \prime \prime} l^{\prime \prime \prime}{ }^{2} L^{\pi}\right\rangle \text {. }
$$

The above notation for the three PCFs applies to both $1 s^{2} 2 s^{2} \mathrm{~S}$ or $1 \mathrm{~s}^{2} 2 \mathrm{p}^{2} \mathrm{P}^{\mathrm{o}}$ states, with $L=l$.

For the first two PCFs (23) and (24), we optimize all the correlation orbitals, freezing the $1 \mathrm{~s}$ and $2 l(=2 \mathrm{~s}$ or $2 \mathrm{p})$ to the HF solution of $1 \mathrm{~s}^{2} 2 \mathrm{~s}^{2} \mathrm{~S}$. This strategy is inadequate for $\Lambda_{1 \mathrm{~s} 1 \mathrm{~s} 2 l}$ since it only contains triple excitations that do not interact with the reference CSF. The optimization of the corresponding orbital set becomes then more tricky. In the previous paper [20], we chose to use a SD-MR to include triple excitations. In the present work, we dedicate a specific PCF to these. We first define an 'extended' SD expansion for a reference set built on the $(n=2,3)$ shells and optimize it by allowing variations in the correlation orbitals only. This expansion opens an indirect interaction between the triple excitations and the reference CSF. For capturing these higher-order effects, we optimize the $n>3$ orbitals during the MR-PCF procedure. The $n=4$ layer is therefore the first one that effectively represents threeelectron excitations. Figure 4 illustrates for the two states that the DPCFI convergence is faster than the traditional CASMCHF approach based on the same CSF expansions. For a given orbital AS, the corresponding total energy value is indeed systematically below the CAS-MCHF result. Since the angular content of the wave function (maximum $l$-value for in the oneelectron basis) is identical for both methods, we conclude that the DPCFI method captures more efficiently electronic correlation for a given atomic system. A similar improvement is a priori expected for any other spectroscopic property. Figure 5 presents the convergence pattern of the contact term for the ground state and the electric quadrupole parameter of the first excited state of neutral lithium. As it clearly appears, the hyperfine parameters are not converging as smoothly as the total energy. It is well known that the relevant expectation values are extremely sensitive to single excitations [31] and it is worthwhile to attempt another approach for treating this excitation family independently. 

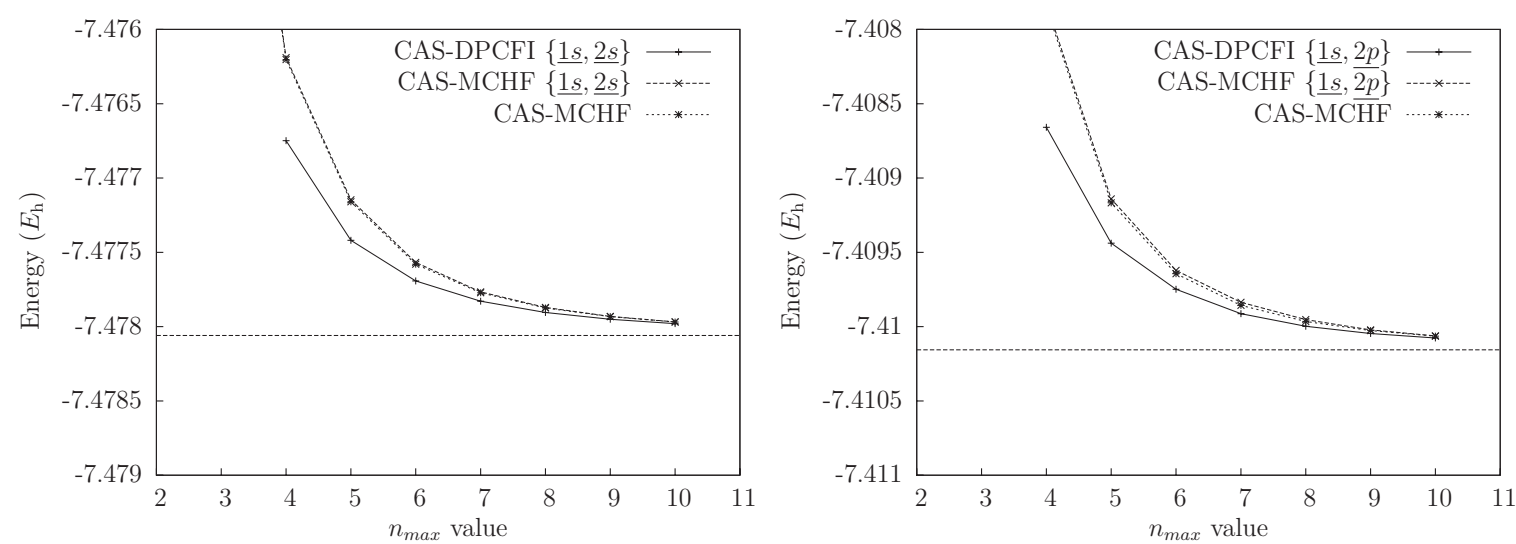

Figure 4. Convergence of the total energy for the ground state $1 s^{2} 2 s^{2} \mathrm{~S}$ of lithium on the left and for the first excited state $1 s^{2} 2 p^{2} \mathrm{P}^{\mathrm{o}}$ on the right. The $n_{\max }$ value specifies the orbital active set ( $n \leqslant n_{\max }$, without any angular limitation). The reference values (dotted lines) correspond to the absolute energy values obtained by Yan et al [41].
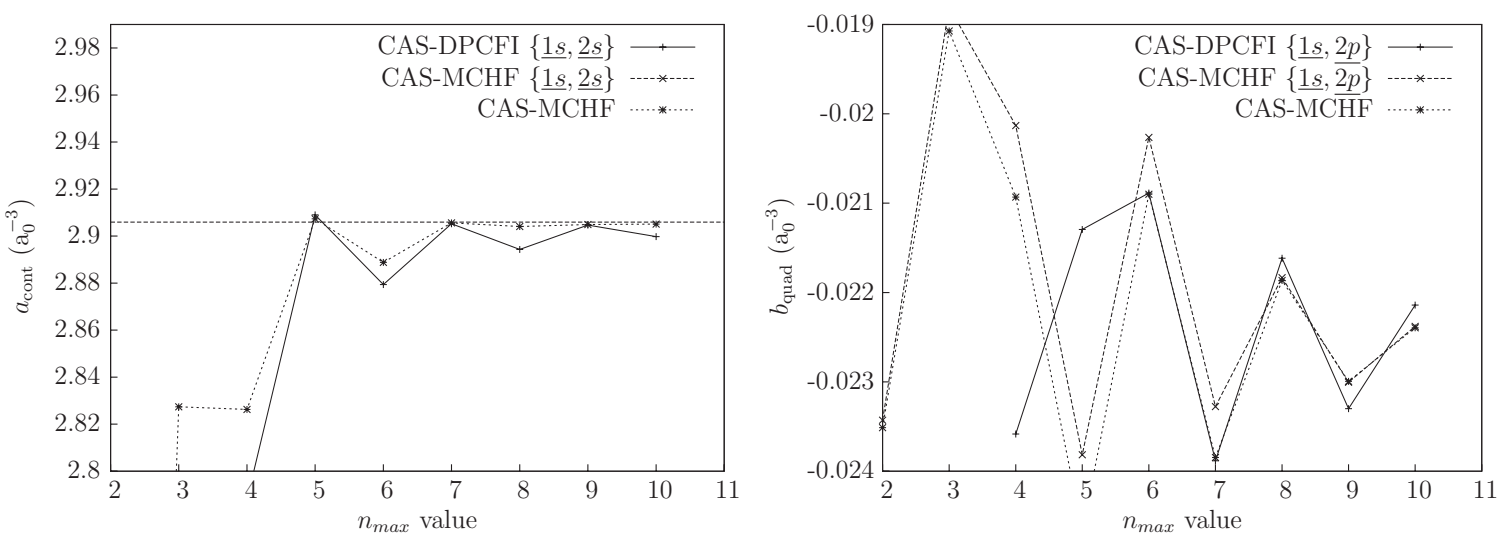

Figure 5. Convergence of the hyperfine contact parameter of the Li ground state (on the left) and of the electric quadrupole hyperfine parameter of the first excited state (on the right). The $n_{\max }$ value specifies the orbital active set ( $n \leqslant n_{\max }$, without any angular limitation). The reference value for the contact $a_{c}$ parameter (dotted line) corresponds to the value obtained by Yan et al [42].

6.3.2. A partitioned correlation function dedicated to corepolarization. For describing more accurately the hyperfine interaction, we split the $\Lambda_{1 \mathrm{~s}-1 \mathrm{~s} 1 \mathrm{~s}} \mathrm{PCF}$ in two subspaces

$$
\Lambda_{1 \mathrm{~s}-1 \mathrm{~s} 1 \mathrm{~s}} \rightarrow \Lambda_{1 \mathrm{~s}}+\Lambda_{1 \mathrm{~s} 1 \mathrm{~s}} .
$$

The $\Lambda_{1 \mathrm{~s}}$ PCF function focusing on the single excitations is dedicated to capture core-polarization effects. The manyelectron wave function is then written as the reference function corrected by four $(p=4)$ different PCFs with their own orbital set: the two first, associated to the CV (24) and to the triple excitations (25), as described in the previous subsection, completed by

- a third one that takes care of the single excitations of the 1s shell

$$
\left|\Lambda_{1 \mathrm{~s}}\right\rangle=\left|1 \mathrm{~s}^{2} 2 l^{2} L^{\pi}\right\rangle+\sum_{n^{\prime} l^{\prime}}\left|1 \mathrm{~s} 2 l n^{\prime} l^{\prime 2} L^{\pi}\right\rangle,
$$

- a last one associated to the double excitations of the $1 \mathrm{~s}$ shell

$$
\left|\Lambda_{1 \mathrm{~s} 1 \mathrm{~s}}\right\rangle=\left|1 \mathrm{~s}^{2} 2 l^{2} L^{\pi}\right\rangle+\sum_{n^{\prime} l^{\prime}, n^{\prime \prime} l^{\prime \prime}}\left|2 l n^{\prime} l^{\prime} n^{\prime \prime} l^{\prime \prime 2} L^{\pi}\right\rangle .
$$

Excitations considered in (27) describe spin-polarization, for both the ${ }^{2} \mathrm{~S}$ and ${ }^{2} \mathrm{P}^{\mathrm{o}}$ states, since single excitations can break the singlet spin coupling between the two core electrons. For the first excited state, these excitations can also break the angular coupling associated with orbital-polarization. It is well known that the hyperfine parameters are sensitive to these excitations [43] and some improvement is expected in their evaluation thanks to the splitting (26). The results are presented in figures $6-8$. By comparing figures 4 and 6 , it is obvious that the decomposition (26) does not affect the total energy value. The DPCFI method still captures correlation more efficiently than the traditional MCHF calculations. The interesting improvement appears for the different hyperfine parameters. Figures 7 and 8 illustrate their progressive convergence, respectively for the ground and the first excited states. The use of the orbital set tailored for capturing the spin- and orbital-CP enhanced beautifully the convergence pattern of all the hyperfine parameters. The resulting trends are much smoother than those of the global core approach and the ordinary MCHF (see figure 5). All oscillations disappeared and we reach reasonably well-converged values around $n=5$. Lithium is a small atom and it is possible to enlarge an ordinary orbital basis to get converged values for all quantities. For larger atoms with more complicated shell structures it is, to set things into perspective, often not possible to extend the radial orbital basis very much due to a rapidly growing number of 

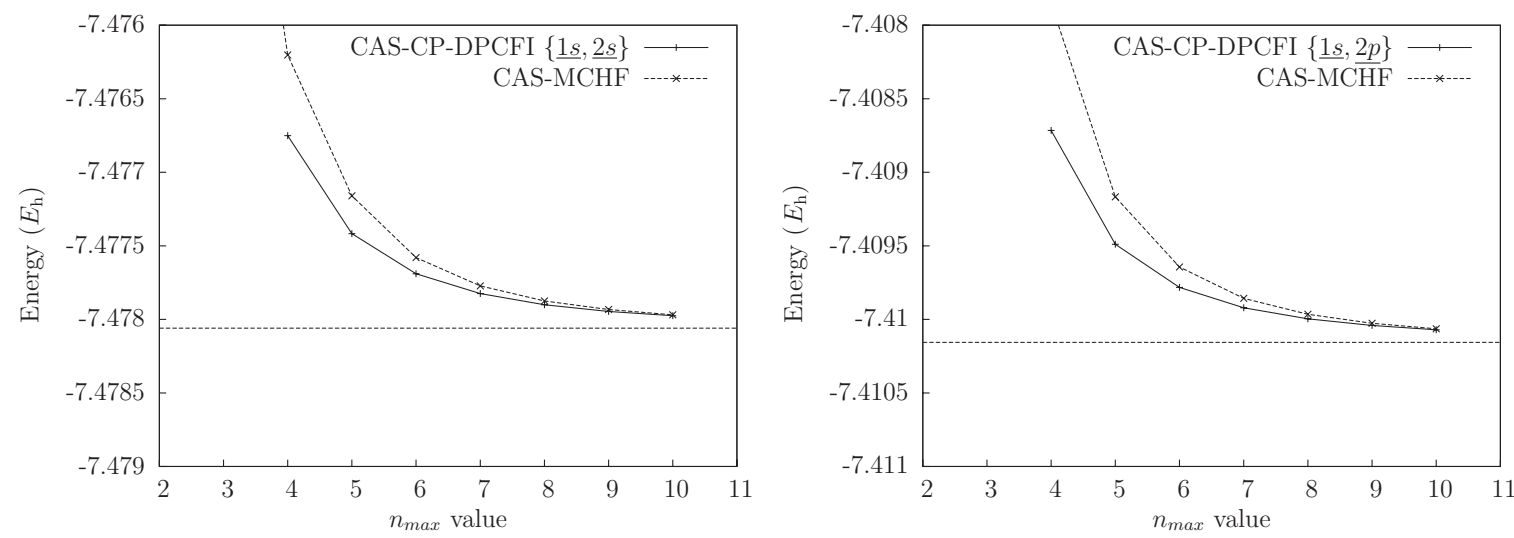

Figure 6. Convergence of the absolute energy for $1 \mathrm{~s}^{2} 2 \mathrm{~s}^{2} \mathrm{~S}$ (left) and $1 \mathrm{~s}^{2} 2 \mathrm{p}^{2} \mathrm{P}^{\mathrm{o}}$ (right) in neutral lithium. The $n_{\max }$ value specifies the orbital active set ( $n \leqslant n_{\max }$, without any angular limitation). The reference values (dotted line) correspond to the results obtained by Yan $e t$ al [41].

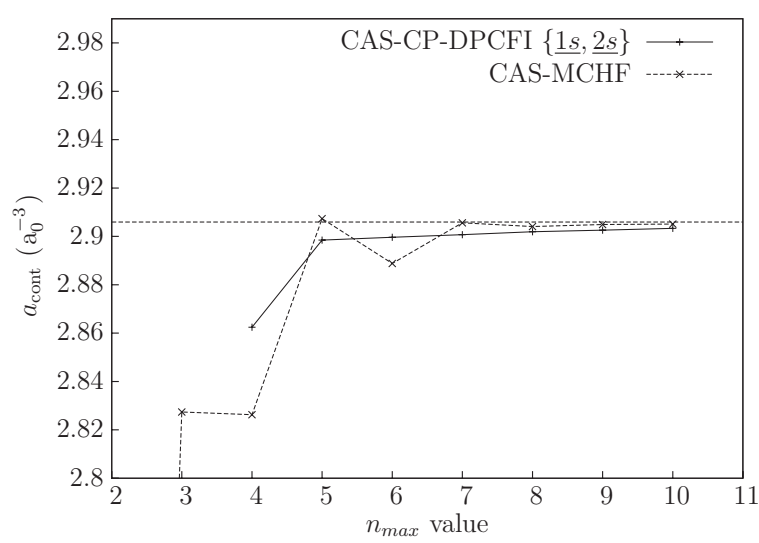

Figure 7. Convergence of the hyperfine contact parameter for the ground state of lithium. The $n_{\max }$ value specifies the orbital active set $\left(n \leqslant n_{\max }\right.$, without any angular limitation). The reference value (dotted line) correspond to the results obtained by Yan et al [42].

Table 2. Energies, $S_{\mathrm{sms}}$ and $a_{\text {cont }}$ for an increasing maximum principal quantum number for the ground state of $\mathrm{Li}$.

\begin{tabular}{clll}
\hline \multicolumn{4}{c}{ CAS-CP-DPCFI $\{\underline{1 \mathrm{~s}}, \underline{2 \mathrm{~s}}\}$} \\
\hline$n_{\max }$ & Energy $\left(E_{\mathrm{h}}\right)$ & $S_{\text {sms }}\left(\mathrm{a}_{0}^{-2}\right)$ & $a_{\text {cont }}\left(\mathrm{a}_{0}^{-3}\right)$ \\
\hline $\mathrm{HF}$ & -7.432726927 & 0.000000000 & 2.0932317 \\
4 & -7.476750919 & 0.304445112 & 2.8624693 \\
5 & -7.477417364 & 0.303171470 & 2.8984561 \\
6 & -7.477689394 & 0.302588400 & 2.8996669 \\
7 & -7.477824739 & 0.302322528 & 2.9007289 \\
8 & -7.477900177 & 0.302162573 & 2.9019278 \\
9 & -7.477946240 & 0.302062836 & 2.9025948 \\
10 & -7.477975286 & 0.302006041 & 2.9033303 \\
{$[41,42]$} & $-7.47806032310(31)$ & $0.301842809(15)$ & $2.905922(50)$ \\
\hline
\end{tabular}

CSFs and here the fast convergence of the CP-DPCFI method, together with the fact that orbital sets for different shells can be optimized independently of each other, represents a major improvement in the general methodology.

The values of the corresponding total energies, $S_{\mathrm{sms}}$ and hyperfine parameters are reported in tables 2 and 3. As in the figures, we compare the DPCFI values with Yan et al $[41,42]$ results using Hylleraas-type variational method. The remaining difference between both studies may be attributed to
Table 3. Energies, $S_{\mathrm{sms}}$ and hyperfine parameters obtained using the PCFI method for the ${ }^{2} \mathrm{P}^{\mathrm{o}}$ state.

\begin{tabular}{cccl}
\hline & \multicolumn{3}{c}{ CAS-CP-DPCFI $\{\underline{1 \mathrm{~s}}, \underline{\mathrm{p}}\}$} \\
\hline$n_{\max }$ & Energy $\left(E_{\mathrm{h}}\right)$ & $S_{\text {sms }}\left(\mathrm{a}_{0}^{-2}\right)$ & $a_{\text {orb }}\left(\mathrm{a}_{0}^{-3}\right)$ \\
\hline $\mathrm{HF}$ & -7.365069658 & -0.041898309 & 0.0585716 \\
4 & -7.408715289 & 0.249999650 & 0.0611674 \\
5 & -7.409489337 & 0.248269629 & 0.0626329 \\
6 & -7.409782422 & 0.247618411 & 0.0627899 \\
7 & -7.409921067 & 0.247249783 & 0.0628740 \\
8 & -7.409996500 & 0.247032302 & 0.0629599 \\
9 & -7.410041507 & 0.246907864 & 0.0629917 \\
10 & -7.410071044 & 0.246830104 & 0.0630317 \\
{$[41]$} & $-7.4101565218(13)$ & $0.24673781(71)$ & \\
\hline$n_{\max }$ & $a_{\text {dip }}\left(\mathrm{a}_{0}^{-3}\right)$ & $a_{\text {cont }}\left(\mathrm{a}_{0}^{-3}\right)$ & $b_{\text {quad }}\left(\mathrm{a}_{0}^{-3}\right)$ \\
\hline $\mathrm{HF}$ & -0.0117143 & 0.0000000 & -0.0234287 \\
4 & -0.0130095 & -0.2078035 & -0.0220013 \\
5 & -0.0133191 & -0.2122187 & -0.0225979 \\
6 & -0.0133551 & -0.2122960 & -0.0226542 \\
7 & -0.0133691 & -0.2128985 & -0.0226978 \\
8 & -0.0133940 & -0.2130121 & -0.0227275 \\
9 & -0.0133960 & -0.2133731 & -0.0227428 \\
10 & -0.0134106 & -0.2134760 & -0.0227578 \\
{$[42]$} & & $-0.214860(50)$ & \\
\hline & & & \\
\hline
\end{tabular}

the slow angular convergence rate $\left(E_{l}-E_{l-1}=\mathcal{O}(l+1 / 2)^{-4}\right)$ of the $\left(1 / r_{12}\right)$ angular development [44].

Table 4 presents the transitions energies, line strengths and weighted oscillator strengths obtained using the CAS-CPDPCFI method. This table illustrates the slow convergence of the oscillator strength in both gauges. It leads to a remaining gap between the length and the velocity gauges for the $n=10$ results. We suspect two effects playing against a fast convergence: (i) the use of frozen spectroscopic orbitals fixed to the HF solution, (ii) the independent optimization of the PCF orbital sets that forbids the coupling between the PCFs subspaces to capture the indirect effects in the orbital optimization. The unconstrained solution could be obtained by solving a general non-orthogonal MCHF problem mixing the different PCFs for the orbital SCF optimization, and substituting, at the end of each orbital SCF step, 

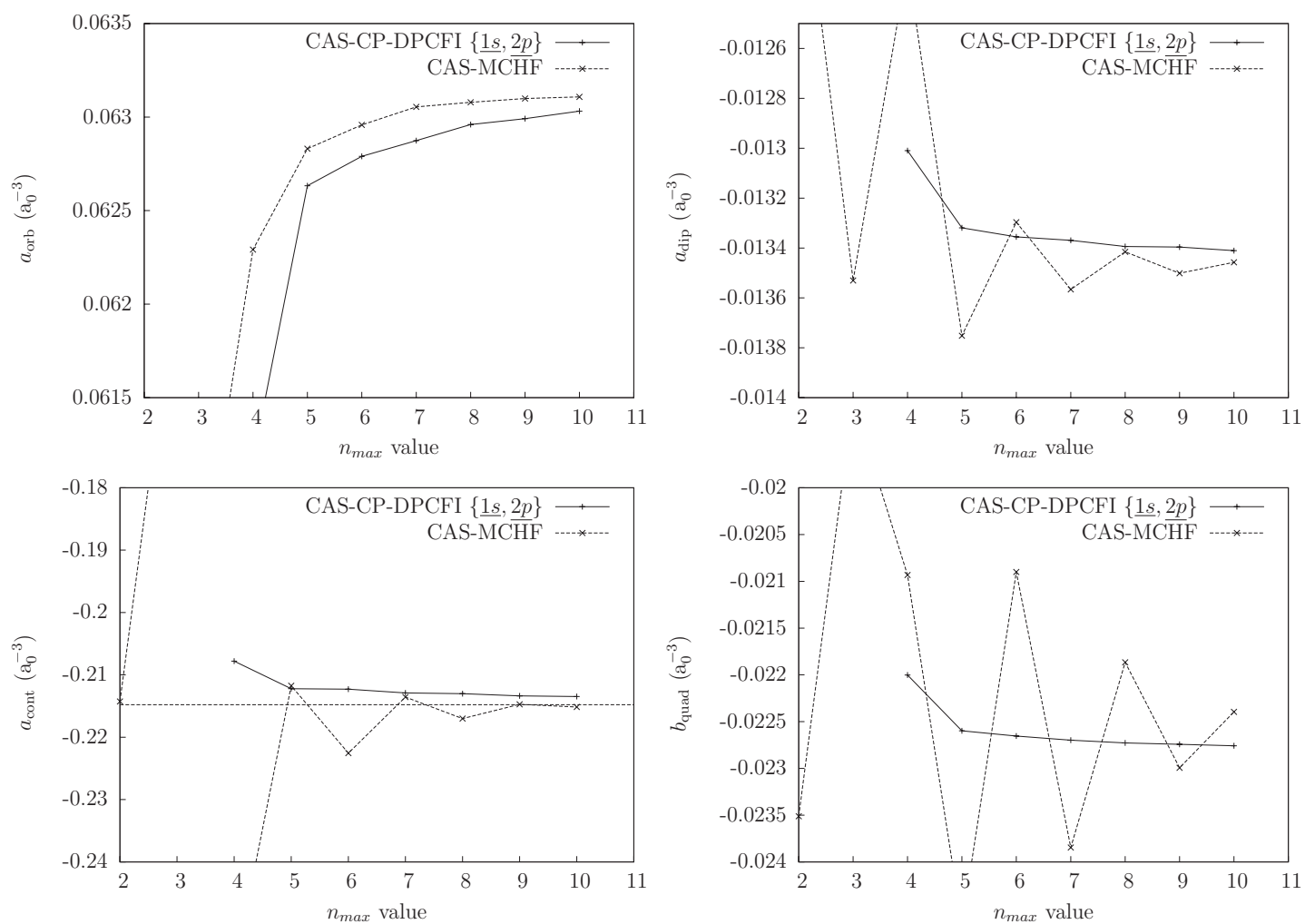

Figure 8. Convergence of the hyperfine parameters for the first excited state, $1 \mathrm{~s}^{2} 2 \mathrm{p}^{2} \mathrm{P}^{\mathrm{o}}$ of lithium. The $n_{\max }$ value specifies the orbital active set $\left(n \leqslant n_{\max }\right.$, without any angular limitation). The reference value (dotted line) corresponds to the result obtained by Yan et al [42].

Table 4. Line strength $\left(S_{l}, S_{v}\right)$ and oscillator strength $\left(g f_{l}, g f_{v}\right)$ for the ${ }^{2} \mathrm{P}^{\mathrm{o}}{ }^{2} \mathrm{~S}$ resonance line.

\begin{tabular}{llllll}
\hline \multicolumn{5}{c}{ CAS-CP-DPCFI $\{\underline{1 \mathrm{~s}}, \underline{2 l}\}$} \\
\hline$n_{\max } \Delta E\left(\mathrm{~cm}^{-1}\right)$ & $S_{l}\left(\mathrm{e}^{2} \mathrm{a}_{0}^{2}\right)$ & $S_{v}\left(\mathrm{e}^{2} \mathrm{a}_{0}^{2}\right)$ & $g f_{l}$ & $g f_{v}$ \\
\hline 4 & 14930.92 & 33.4862 & 32.7741 & 1.51872 & 1.48642 \\
5 & 14907.31 & 33.1284 & 32.9457 & 1.50011 & 1.49184 \\
6 & 14902.69 & 33.0804 & 32.9798 & 1.49748 & 1.49292 \\
7 & 14901.97 & 33.0639 & 32.9835 & 1.49666 & 1.49302 \\
8 & 14901.97 & 33.0536 & 32.9761 & 1.49619 & 1.49268 \\
9 & 14902.20 & 33.0465 & 32.9707 & 1.49589 & 1.49246 \\
10 & 14902.09 & 33.0414 & 32.9692 & 1.49565 & 1.49238 \\
$\mathrm{CAS}(n=10)$ & 14902.23 & 33.0038 & 32.9973 & 1.49396 & 1.49367 \\
$\mathrm{CAS}(n=10)\{\underline{1 \mathrm{~s}}, \underline{2 l}\}$ & 14902.48 & 33.0075 & 32.9885 & 1.49415 & 1.49329 \\
{$[45]$} & $14903.16176(29)$ & 33.00066933 & 33.00081733 & 1.4939139 & 1.4939206 \\
\hline
\end{tabular}

the conventional CI by a DPCFI (i.e. non-orthogonal CI) calculation to get the desired deconstrained eigenvector.

\section{Partial deconstraining schemes}

It is worthwhile to investigate how indirect effects are distributed over CSFs. To answer this question, we focus our analysis on the mixing coefficient constraint itself by eliminating the impact of the orbital optimization. We are therefore diagonalizing the Hamiltonian operator within each PCF space, adopting the same orthonormal orbital common set for all PCFs. At each step we promote one selected CSF, included in one PCF, at the same level than the reference $\mathrm{CSF}$ and then we solve the associated eigenvalue problem for finding the total energy and the two other properties. In this way, we progressively remove all constraints in the expansion coefficients, going from a low-dimension PCFI to a larger CSF-CI calculation. Our selection rule for choosing at each step the promoted CSF is somewhat arbitrary since we adopt the order of the configuration list produced by lsgen program [46]. In the present case, the hierarchy follows (i) $\mathrm{S}$ from the valence, (ii) $\mathrm{S}$ from the core, (iii) $\mathrm{D}$ from $\mathrm{CV}$ and (iv) D from the core. Figure 9 illustrates the evolution, for each property, of the relative difference between the value calculated for a given matrix size, associated with a partially deconstrained many-electron wave function, and the corresponding CSF-CI result. Figure 9 shows not only that the operators are affected differently by the constraint, but 

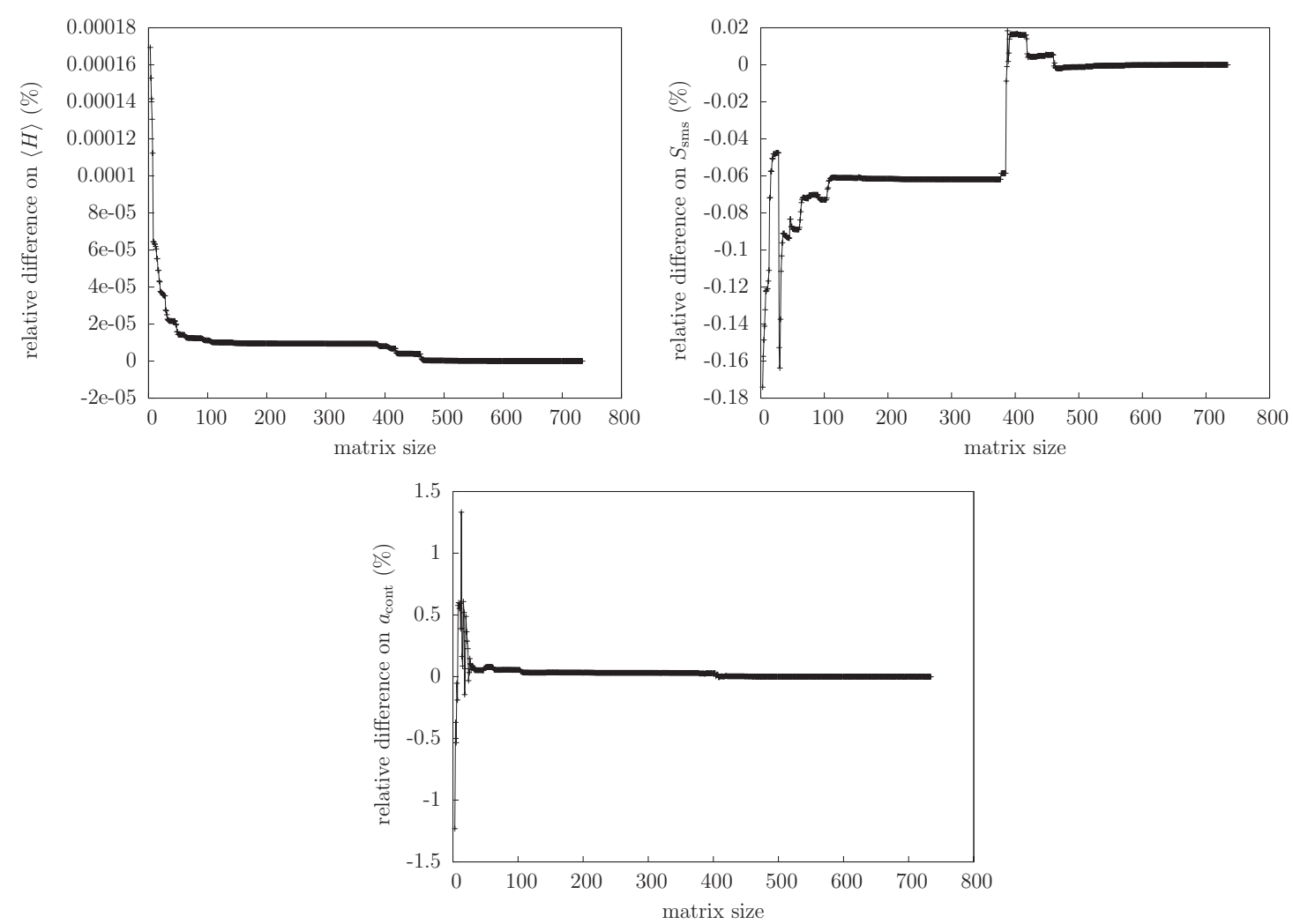

Figure 9. Progressive deconstraint of the many-electron wave function. The three curves illustrate the relative difference between the CI calculation and the partially deconstrained problem for respectively the total energy $\langle H\rangle$, the specific mass shift parameter $\left(S_{\mathrm{sms}}\right)$ and the hyperfine contact parameter $\left(a_{\text {cont }}\right)$ for the ground state $1 \mathrm{~s}^{2} 2 \mathrm{~s}^{2} \mathrm{~S}$ of neutral lithium.

also that the way in which the constraint effect is distributed over the CSFs directly depends on the selected operator itself. One may note that, accordingly to the variational principle, the total energy is monotonically decreasing until it reaches the CI value, but the evolution is much more erratic for the two other properties. The presence of many plateaus in the graphs of figure 9 suggests that some efficient partial deconstraint scheme might be found. For the contact term, the latter is accidentally revealed, as illustrated by the third graph of figure 9, due to the fact that the most important contributions arise from the single excitations from the core that appear first in the generated list. For the $S_{\text {sms }}$ parameter, the jump occurring around 400 corresponds to the presence of $2 \mathrm{~s} 2 \mathrm{p}^{2}{ }^{2} \mathrm{~S}$ in the expansion of $\Lambda_{1 \mathrm{~s}-1 \mathrm{~s} 1 \mathrm{~s}}$. This excitation is introduced quite late in the sequence since it is generated by lsgen in the fourth category (double excitations from the core).

By selecting and deconstraining the CSFs which constitute the dominant contributors to the constraint effect, it should be possible to optimize the CSF space partition that produces the more efficient PCF basis. We will show that full deconstraining is not always necessary and that some deconstraining schemes might be better than others. The key point in this analysis is to find in the CF space, for a given property, the best candidates for a possible promotion in the CSF transfer (17). As illustrated by figure 9, it is hard to predict the behaviour of a given property with respect to a particular scheme of deconstraint, except for the total energy, thanks to the Hylleraas-Undheim theorem [47]. It is here interesting to remember what we learn from the timeindependent perturbation theory. Defining the $\left|n^{(0)}\right\rangle$ states as the eigenstates of the zeroth-order Hamiltonian $H^{(0)}$, and introducing a perturbation $V$ such as $H=H^{(0)}+\lambda V$, the eigenfunction $|n\rangle$ of $H$ can be expressed as the following $\lambda$-expansion:

$$
\begin{aligned}
|n\rangle & =\left|n^{(0)}\right\rangle+\lambda \sum_{k \neq n}\left|k^{(0)}\right\rangle \frac{V_{k n}}{E_{n}^{(0)}-E_{k}^{(0)}} \\
& +\lambda^{2}\left(\sum_{k \neq n} \sum_{l \neq n} \frac{\left|k^{(0)}\right\rangle V_{k l} V_{l n}}{\left(E_{n}^{(0)}-E_{k}^{(0)}\right)\left(E_{n}^{(0)}-E_{l}^{(0)}\right)}\right. \\
& \left.-\sum_{k \neq n} \frac{\left|k^{(0)}\right\rangle V_{n n} V_{k n}}{\left(E_{n}^{(0)}-E_{k}^{(0)}\right)^{2}}\right)+\cdots
\end{aligned}
$$

where the $V_{i j}=\left\langle i^{(0)}|V| j^{(0)}\right\rangle$ matrix elements are calculated using the zero-order basis [48]. From this expression, it is easy to derive the $\lambda$-expansion of the expectation value of any operator $X$ :

$$
\begin{aligned}
& \langle n|X| n\rangle=\left\langle n^{(0)}|X| n^{(0)}\right\rangle \\
& +\lambda\left(\sum_{k \neq n} \frac{X_{n k} V_{k n}}{E_{n}^{(0)}-E_{k}^{(0)}}+\sum_{k \neq n} \frac{X_{k n} V_{k n}}{E_{n}^{(0)}-E_{k}^{(0)}}\right) \\
& \quad+\lambda^{2}\left(\sum_{k \neq n} \sum_{k^{\prime} \neq n} \frac{X_{k k^{\prime}} V_{k n} V_{k^{\prime} n}}{\left(E_{n}^{(0)}-E_{k}^{(0)}\right)\left(E_{n}^{(0)}-E_{k^{\prime}}^{(0)}\right)}\right. \\
& +\sum_{k \neq n} \sum_{l \neq n} \frac{X_{n k} V_{k l} V_{l n}}{\left(E_{n}^{(0)}-E_{k}^{(0)}\right)\left(E_{n}^{(0)}-E_{l}^{(0)}\right)}-\sum_{k \neq n} \frac{X_{n k} V_{n n} V_{k n}}{\left(E_{n}^{(0)}-E_{k}^{(0)}\right)^{2}}
\end{aligned}
$$


Table 5. The first ten more important contributions to (31) for the lithium ground state $S_{\text {sms }}$ parameter.

\begin{tabular}{cllr}
\hline & $\Phi_{i}$ & $\Phi_{j}^{\prime}$ & $2\left\langle\Phi_{i}\left|X_{\mathrm{sms}}\right| \Phi_{j}\right\rangle$ \\
\hline 1. & $1 \mathrm{~s}^{2} 2 \mathrm{~s}$ & $2 \mathrm{~s} 2 \mathrm{p}^{2}\left({ }^{1} \mathrm{~S}\right)$ & 0.31761489 \\
2. & $2 \mathrm{~s} 2 \mathrm{p}^{2}\left({ }^{1} \mathrm{~S}\right)$ & $2 \mathrm{~s} 3 \mathrm{~s}$ & -0.01267429 \\
3. & $1 \mathrm{~s}^{2} 2 \mathrm{~s}$ & $1 \mathrm{~s} 2 \mathrm{p}\left[{ }^{1} \mathrm{P}\right] 4 \mathrm{p}$ & 0.01141852 \\
4. & $2 \mathrm{~s} 2 \mathrm{p}^{2}$ & $2 \mathrm{~s} 3 \mathrm{~d}^{2}\left({ }^{1} \mathrm{~S}\right)$ & -0.00580568 \\
5. & $2 \mathrm{~s} 3 \mathrm{~s}^{2}$ & $2 \mathrm{~s} 3 \mathrm{p}^{2}\left({ }^{1} \mathrm{~S}\right)$ & -0.00397443 \\
6. & $1 \mathrm{~s}^{2} 2 \mathrm{~s}$ & $2 \mathrm{~s} 2 \mathrm{p}\left[{ }^{3} \mathrm{P}\right] 3 \mathrm{p}$ & -0.00342031 \\
7. & $1 \mathrm{~s}^{2} 2 \mathrm{~s}$ & $2 \mathrm{~s} 3 \mathrm{p}^{2}\left({ }^{1} \mathrm{~S}\right)$ & 0.00154986 \\
8. & $1 \mathrm{~s}^{2} 2 \mathrm{~s}$ & $2 \mathrm{~s} 2 \mathrm{p}\left[{ }^{1} \mathrm{P}\right] 3 \mathrm{p}$ & -0.00111510 \\
9. & $2 \mathrm{~s} 3 \mathrm{p}^{2}\left({ }^{1} \mathrm{~S}\right)$ & $2 \mathrm{~s} 3 \mathrm{~d}^{2}\left({ }^{1} \mathrm{~S}\right)$ & -0.00110056 \\
10. & $2 \mathrm{~s} 2 \mathrm{p}\left[{ }^{3} \mathrm{P}\right] 3 \mathrm{p}$ & $2 \mathrm{~s}(1) 3 \mathrm{~s}(2)$ & 0.00109413 \\
11. & $\cdots$ & $\ldots$ & $\ldots$ \\
\hline
\end{tabular}

$$
\begin{aligned}
& +\sum_{k \neq n} \sum_{l \neq n} \frac{X_{k n} V_{k l} V_{l n}}{\left(E_{n}^{(0)}-E_{k}^{(0)}\right)\left(E_{n}^{(0)}-E_{l}^{(0)}\right)} \\
& \left.-\sum_{k \neq n} \frac{X_{k n} V_{n n} V_{k n}}{\left(E_{n}^{(0)}-E_{k}^{(0)}\right)^{2}}\right)+\cdots
\end{aligned}
$$

where $X_{i j}=\left\langle i^{(0)}|X| j^{(0)}\right\rangle$. The first term is the reference value. The $\mathcal{O}(\lambda)$ terms are called the 'crossed second-order' (cso) corrections to the zeroth-order expectation value $\langle X\rangle_{n}$ since they involve both the Hamiltonian and the operator $X$ in the coupling matrix elements. These cso contributions have been used successfully in the parametric method to analyse isotope shifts [49], hyperfine structures [50] and field shifts [51]. From this expression, one can realize that it would be interesting for an efficient $a b$ initio approach, to partially deconstrain the PCFs according to their contribution to the expectation value. The CSFs that are the best candidates for a transfer promotion (17) are the ones that are coupled to the reference by both the Hamiltonian and the relevant operator $\left(X_{n k} \neq 0 ; V_{n k} \neq 0\right)$ but these are not the only ones. For the SMS parameter of the lithium ground state, the HF value is strictly zero. The non zero value is made of correlation components only

$$
S_{\mathrm{sms}}=\sum_{i j} c_{i} c_{j}\left\langle\Phi_{i}\left|X_{\mathrm{sms}}\right| \Phi_{j}\right\rangle=0.301450504
$$

and a close ranking analysis of the SMS matrix shows that the first ten contributions to (31) reported in table 5 account for $95 \%$ of the total contributions.

In a perturbation approach, the contributions appearing in lines $(1,3,6,7,8)$ of table 5 would be cso contributions

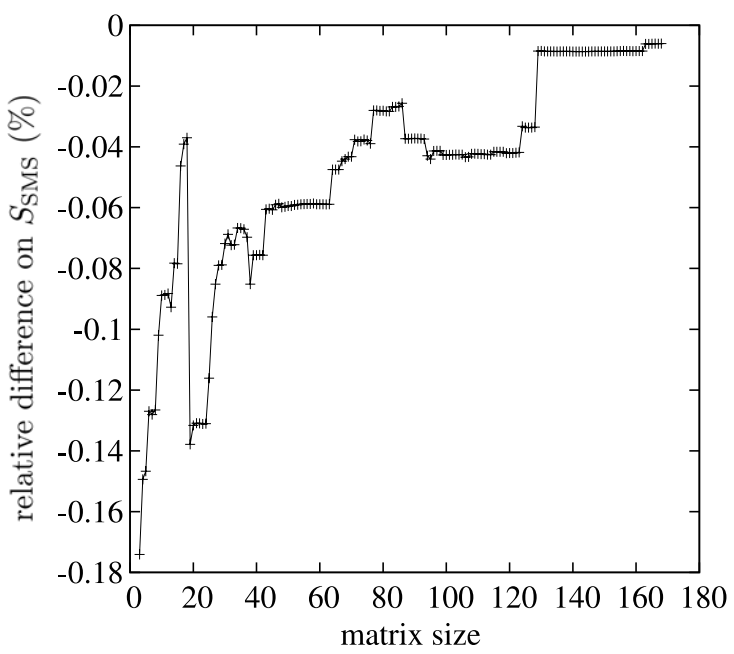

Figure 10. Li $1 \mathrm{~s}^{2} 2 \mathrm{~s}^{2} \mathrm{~S}$. Partial deconstraint according to the SMS contributions sorting.

of $\mathcal{O}(\lambda)$. The other five higher-order corrections are rather important too and would result from contributions of the type $\left(X_{k k^{\prime}} V_{k n} V_{k^{\prime} n}\right)$. If we use, for the progressive PCF deconstraint, the list of the CSFs sorted according to their contribution to the $S_{\text {sms }}$ parameter we get a very efficient deconstraining scheme for this property. Figure 10 illustrates indeed that the transfer of the $\simeq 140$ first CSFs produces a rather good result, to put in contrast with figure 9.

For the total energy, the most natural deconstraining scheme is based on the weight criteria. The importance of a CSF is indeed mainly determined by the magnitude of its mixing coefficient that can be estimated from the first-order correction to the wave function (the $\mathcal{O}(\lambda)$ term in (29)), i.e.

$$
c_{k} \approx \frac{H_{k n}}{E_{n}^{(0)}-E_{k}^{(0)}} .
$$

To illustrate the gain of partial deconstraint, we focus on Be $1 s^{2} 2 s 2 p^{1} P^{o}$ considered in section 6.2. Starting from the constrained representation corresponding to the PCFI solution, we deconstrain, in each of the three MR-PCF wave function (12), all CSFs having a mixing coefficient larger than a given threshold, i.e. $\left|c_{j}^{\Lambda}\right| \geqslant \epsilon$. Repeating this operation for a decreasing threshold $\epsilon$, we progressively move from the constrained PCFI solution $(\epsilon=1)$ to the deconstrained DPCFI one $(\epsilon=0)$. Using $\epsilon=1 \times 10^{-4}$, we reduce the computer time by a factor of 10-20 relatively to the DPCFI calculations.

Table 6. Comparison between constrained PCFI, partially deconstrained $\left(\epsilon=1.10^{-4}\right)$ and DPCFI results for Be $1 \mathrm{~s}^{2} 2 \mathrm{~s} 2 \mathrm{p}^{1} \mathrm{P}^{\mathrm{o}} . E$, $S_{\mathrm{sms}}, a_{\mathrm{orb}}$ and $b_{\text {quad }}$ are respectively the total energy, the specific mass shift parameter and the hyperfine parameters as defined in [30].

\begin{tabular}{lrrrrr}
\hline & $n \leqslant$ & \multicolumn{1}{c}{$E\left(E_{\mathrm{h}}\right)$} & \multicolumn{1}{c}{$S_{\text {sms }}\left(\mathrm{a}_{0}^{-2}\right)$} & $a_{\text {orb }}\left(\mathrm{a}_{0}^{-3}\right)$ & \multicolumn{1}{c}{$b_{\text {quad }}\left(\mathrm{a}_{0}^{-3}\right)$} \\
\hline HF & 2 & -14.394735414 & 0.004136928 & 0.0917535 & -0.0367014 \\
MR & 3 & -14.421696066 & -0.000135774 & 0.1563725 & -0.0590602 \\
SD-MR-MCHF & 10 & -14.473005606 & 0.434722502 & 0.1846345 & -0.0672910 \\
& 7 & -14.472842258 & 0.434834517 & 0.1837046 & -0.0667457 \\
Partially & 8 & -14.473018319 & 0.434557950 & 0.1838054 & -0.0656012 \\
Deconstrained SD-MR-PCFI & 9 & -14.473117735 & 0.434469982 & 0.1839501 & -0.0659210 \\
& 10 & -14.473176238 & 0.434340871 & 0.1839869 & -0.0660065 \\
SD-MR-PCFI & 10 & -14.473110086 & 0.439248058 & 0.1769675 & -0.0643341 \\
SD-MR-DPCFI & 10 & -14.473185865 & 0.434103182 & 0.1844820 & -0.0675441 \\
\hline
\end{tabular}


For the $n=10 \mathrm{AS}$, the size of the eigenvalue problem is respectively 9 (PCFI), 788 (partially deconstrained) and 21632 (DPCFI) wave functions. The values for the total energy, the $S_{\text {sms }}$ and hyperfine parameters are compared with the HF, MR, $(n=10)$ SD-MR-MCHF, PCFI and DPCFI results in table 6 . The $(n=10)$ PCFI result is already better than the ( $n=10)$ SD-MR-MCHF value for the total energy. Taken the DPCFI results as the reference for the other parameters, decontraction becomes important as discussed in section 4 but partial deconstraint with $\epsilon=1 \times 10^{-4}$ allows to produce quite accurate results, recapturing $96 \%\left(S_{\mathrm{sms}}\right), 93 \%\left(a_{\text {orb }}\right)$ and $52 \%$ $\left(b_{\text {quad }}\right)$ of the way to the DPCFI values. The fact that only $52 \%$ of $b_{\text {quad }}$ is recaptured once again illustrates the weak coupling of this quantity to the energy.

\section{Conclusions and perspectives}

We extend the multiconfiguration Hartree-Fock method by partitioning the correlation function space into several subspaces to get a better description of the dynamical correlation. For each of the subspaces, we optimize a partition correlation function to correct the multireference wave function. The atomic state is described by the eigenstate of a low-dimensional PCFI eigenvalue problem, regrouping the different PCFs with the multireference function. By relaxing the orthonormality restriction on the orbital basis between the different subspaces, one obtains several mutually nonorthogonal orbital basis sets that are better adapted to the short range nature of the dynamical correlation.

Our original work [20], targeting the ground state of beryllium, leads to some unexpected complication when the PCFI eigenvector is used for the calculation of spectroscopic properties other than the total energy. We identified the source of the problem as the loss of variational freedom in solving the interaction eigenvalue problem that determines the PCF weights. We evaluated the differences in terms of convergence between properties calculated, on the one hand with a single orthonormal basis spanning an MCHF wave function in the traditional approach, and on the other hand with several tailored orbital basis sets optimized for different PCFs and underlying a (D)PCFI wave function. In addition to energy we consider specific mass shift, hyperfine structure parameters and transition data. We demonstrate that by (partially) deconstraining the mixing coefficients within each PCF subspace, one converges to the correct limits and keeps the tremendous advantage of improved convergence rates that comes from the use of several orbital sets. Reducing ultimately each PCF to a single CSF with its own orbital basis leads to a non-orthogonal configuration interaction approach.

We found that partial deconstraining schemes are very attractive. For the energy indeed, the PCFI approach is shown to be highly efficient by producing accurate eigenvalues. Partial deconstraining schemes based on the CSF weight criteria appear efficient and natural for the Hamiltonian. For other properties, we demonstrated that a deconstraint scheme based on the ranking of the contributions to the relevant observable becomes much more efficient, as expected from a perturbation analysis. The PCFI variational method, assisted by such a perturbation analysis to define the CSFs that should be targeted in the deconstraining strategy, is extremely promising in view of the present results. The interest of the method is the fast convergence of the desired observable combined with a large reduction factor in the size of the interaction problem (and the expected CPU time gain factor that goes with it), added to the possibility of parallelizing the PCF-optimization processes before regrouping the various subspaces with the reference function to build the atomic state.

The (D)PCFI method is also highly flexible. Taking advantage of the possibility of tailoring the correlation subspace partitions, we built a specific partition correlation function for capturing core-polarization effects. The addition of this PCF tremendously improves the convergence of all the hyperfine parameters. This approach provides values of the total energy, specific mass shift and hyperfine parameters with an acceptable accuracy for rather small $(n=6)$ orbital active sets. These calculations demonstrate that it becomes easy to gain freedom for capturing effects weakly connected to energy, improving the convergence of atomic properties other than the energy.

For the three- and four-electron systems considered in the present work, the (D)PCFI method might not appear as really profitable since rather complete MCHF calculations with a single orthonormal orbital basis can be still performed. For larger systems however, as explained in the introduction, the scaling wall becomes the real barrier and there is no hope of capturing the dynamic correlation between electrons in all the different shells. The breaking down of the very large calculations based on a common orbital set into a series of smaller parallel calculations will then become really relevant and opens new perspectives. With this respect, five- and sixelectron systems that are beyond the current limits of the Hylleraas method are the first targets in our research list.

The proposed (D)PCFI method can be applied to any multiconfiguration approach. We will soon start to implement it in the fully relativistic codes [52] but the present calculations in the non-relativistic framework [53] remain the ideal benchmarks for developing new computational strategies. Another natural line of research is to investigate how the remaining constraints on radial orbitals could be fully relaxed. This long term project could lead to a more general non-orthogonal MCHF approach corresponding to the unconstrained SCF-DPCFI approach.

In quantum chemistry, a method for a one-component relativistic treatment of molecular systems using a multiconfigurational approach (CASSCF) with dynamical correlation treated with second order perturbation theory (CASPT2) has been proposed by Malmqvist et al [55]. In their work, the Hamiltonian matrix is obtained by an extension of the restricted active space state interaction (RASSI) method. They also use non-orthogonal orbitals and open some interesting perspectives that we might investigate for future work, combining RASSI and the present PCFI approach.

\section{Acknowledgments}

SV had an FRIA fellowship from the FRS-FNRS Fund for Scientific Research. MG thanks the Communauté française 
of Belgium (Action de Recherche Concertée) and the Belgian National Fund for Scientific Research (FRFC/IISN Convention) for financial support. Financial support by the Swedish Research Council is gratefully acknowledged.

\section{Appendix A. The PCF building rules for a unit PCFs overlap matrix}

Let us assume that the MR function is built on the reference orthonormal subset $\left\{\phi_{k}\right\}$ :

- $\left|\Psi^{\mathrm{MR}}\right\rangle ;\left\{\phi_{1}, \ldots, \phi_{n}\right\}$

and consider two different PCFs, $\Lambda_{1}$ and $\Lambda_{2}$, built on the MR orbitals and on independent one-electron orbital sets $\left\{\theta_{k}\right\}$ and $\left\{\zeta_{k}\right\}$

- $\left|\Lambda_{1}\right\rangle$ using an orthonormal orbital basis $\left\{\phi_{1}, \ldots, \phi_{n} ; \theta_{1}, \ldots, \theta_{m_{1}}\right\}$,

- $\left|\Lambda_{2}\right\rangle$ using another orthonormal orbital basis $\left\{\phi_{1}, \ldots, \phi_{n} ; \zeta_{1}, \ldots, \zeta_{m_{2}}\right\}$,

both built from excitations of the (multi-)reference function $\Psi^{\mathrm{MR}}$. In our approach, the reference orbitals subsets $\{\phi\}$ of both PCFs $\Lambda_{1}$ and $\Lambda_{2}$ are necessarily identical, being taken from the optimization of the reference function $\left(\Psi^{\mathrm{MR}}\right)$.

In second quantization, one can write the orbital orthonormality within the three subsets $\{\phi\},\{\theta\}$ and $\{\zeta\}$ as follows:

$$
\begin{gathered}
\left\{\phi_{a}, \phi_{b}\right\}=\left\{\phi_{a}^{\dagger}, \phi_{b}^{\dagger}\right\}=0 \quad \text { and } \quad\left\{\phi_{a}, \phi_{b}^{\dagger}\right\}=\delta_{a, b} \\
\left\{\theta_{a}, \theta_{b}\right\}=\left\{\theta_{a}^{\dagger}, \theta_{b}^{\dagger}\right\}=0 \quad \text { and } \quad\left\{\theta_{a}, \theta_{b}^{\dagger}\right\}=\delta_{a, b} \\
\left\{\zeta_{a}, \zeta_{b}\right\}=\left\{\zeta_{a}^{\dagger}, \zeta_{b}^{\dagger}\right\}=0 \quad \text { and } \quad\left\{\zeta_{a}, \zeta_{b}^{\dagger}\right\}=\delta_{a, b} .
\end{gathered}
$$

The orbital orthogonality being imposed within each PCF, we also have

$$
\left\{\phi_{a}, \theta_{b}^{\dagger}\right\}=\left\{\phi_{a}, \zeta_{b}^{\dagger}\right\}=0 .
$$

On the other hand, the orthogonality between $\{\theta\}$ and $\{\zeta\}$ orbital subsets is lost:

$$
\left\{\theta_{a}, \zeta_{b}\right\}=\left\{\theta_{a}^{\dagger}, \zeta_{b}^{\dagger}\right\}=0 \quad \text { with } \quad\left\{\theta_{a}, \zeta_{b}^{\dagger}\right\}=S_{a, b}^{\theta \zeta} .
$$

An arbitrary PCF can be written as a linear combination of CSFs and consequently, of Slater determinants (Sdets). We will show that the overlap between any pair of Sdets appearing in the overlap matrix element involving two different PCFs is zero. A corollary is that the overlap between PCFs is inevitably zero, i.e. $\left\langle\Lambda_{i} \mid \Lambda_{j}\right\rangle=\delta_{i j}$.

Let

- $\langle 0| \phi_{1} \ldots \phi_{n} \ldots \theta_{1} \ldots \theta_{n}$, an arbitrary Sdet belonging to PCF $\left\langle\Lambda_{1}\right|$,

- $\zeta_{n}^{\dagger} \ldots \zeta_{1}^{\dagger} \ldots \phi_{n}^{\dagger} \ldots \phi_{1}^{\dagger}|0\rangle$, another Sdet belonging to the other $\mathrm{PCF}\left|\Lambda_{2}\right\rangle$.

Let us assume that the PCFs are built according the following rules:

(i) the excitation prototypes appearing in different PCFs have necessarily different occupation numbers for the reference orbitals $\left\{\phi_{k}\right\}$

(ii) there is no CSF redundancy $\left(\Lambda_{i} \cap \Lambda_{j}=\varnothing\right)$, (iii) the orthogonality constraints between the variational orbitals, $\left\{\theta_{k}\right\}$ in $\Lambda_{1}$, and $\left\{\zeta_{k}\right\}$ in $\Lambda_{2}$, and the (frozen) reference ones $\left\{\phi_{k}\right\}$ are imposed as usual, through the Lagrange multipliers of the MCHF equations.

By respecting these 'building rules' for PCFs constructed from excitations of orbitals $\left\{\phi_{k}\right\}$ spanning $\Psi^{\mathrm{MR}}$, we are assured that Sdets belonging to different PCFs have different occupation numbers for the reference orbitals $\left\{\phi_{k}\right\}$. This implies that at least one of the creation operators associated with a reference spin-orbital appears only in one of the two Sdets, and not in the other one. This creation operator is then free to 'sail' in the creation/annihilation operator sea, with a possible phase factor as the only price to pay. Moving it to the left-/right-hand side until it acts on the bra/ket vacuum state, we ultimately get a null result.

As an illustration, if the $\phi_{2}$ spin-orbital does not appear in the bra, we have :

$$
\begin{aligned}
& \left\langle 0\left|\phi_{1} \phi_{3} \ldots \phi_{n} \ldots \theta_{1} \ldots \theta_{n} \zeta_{n}^{\dagger} \ldots \zeta_{1}^{\dagger} \ldots \phi_{n}^{\dagger} \ldots \phi_{2}^{\dagger} \phi_{1}^{\dagger}\right| 0\right\rangle \\
& \quad=(-1)^{N_{p}}\left\langle 0\left|\phi_{2}^{\dagger} \phi_{1} \phi_{3} \ldots \phi_{n} \ldots \theta_{1} \ldots \theta_{n} \zeta_{n}^{\dagger} \ldots \zeta_{1}^{\dagger} \ldots \phi_{n}^{\dagger} \ldots \phi_{1}^{\dagger}\right| 0\right\rangle \\
& \quad=0
\end{aligned}
$$

where $N_{p}$ is the required number of permutations.

We have demonstrated that the PCFs overlap matrix is the unit matrix if we respect the above PCF building rules. There are however situations for which violating these rules might be interesting from the variational point of view. In these cases, the PCF overlap matrix is not the unity matrix anymore, forcing us to treat the PCFI interaction problem as the generalized eigenvalue problem (14).

The non-diagonal PCF overlap matrix element $\left\langle\Lambda_{1} \mid \Lambda_{2}\right\rangle$ will indeed differ from zero if

- there exist two CSFs, belonging respectively to $\Lambda_{1}$ and $\Lambda_{2}$, that have identical occupation numbers for all the reference orbitals,

- the reference orbitals cannot be strictly qualified as 'reference' ones, when another subset is used for one of the PCFs i.e. $\left\{\phi_{k}^{\prime}\right\} \neq\left\{\phi_{k}\right\}$. For example, if the $\left\{\phi_{k}^{\prime}\right\}$ orbitals of $\Lambda_{1}$ do not have the same radial functions than the $\left\{\phi_{k}\right\}$ of $\Psi^{\mathrm{MR}}$ and $\Lambda_{2}$, although having the same labels, some commutation rules are lost:

$$
\left\{\phi_{a}^{\prime}, \zeta_{b}^{\dagger}\right\}=S_{a b}^{\phi^{\prime} \zeta}
$$

- non-orthogonalities are introduced within a given PCF, for instance within $\Lambda_{2}$ :

$$
\left\{\phi_{a}, \zeta_{b}^{\dagger}\right\}=S_{a b}^{\phi \theta}
$$

\section{Appendix B. The weight matrix formalism}

In the present appendix, we will show how the whole sub-matrix block of the matrix (15) can be evaluated by performing a single biorthonormal transformation treating simultaneously the counter-transformation of all the elements constituting the block-basis. For this purpose, we introduce the weight matrix $\mathbf{D}^{i}$, corresponding to the deconstrained PCF 
$\left\{\Phi_{1}^{i}, \ldots, \Phi_{h_{i}}^{i}, \bar{\Lambda}_{i}^{d}\right\}$. Each column of the latter is composed of the CSF expansion coefficients for each of the $\left(h_{i}+1\right)$ elements

$$
\mathbf{D}^{i}=\left(\begin{array}{cccc}
1 & & 0 & 0 \\
0 & \ddots & 1 & 0 \\
0 & & 0 & d_{\left(h_{i}+1\right)} \\
& \vdots & & \vdots \\
0 & & 0 & d_{\operatorname{dim}\left(\bar{\Lambda}_{i}\right)} \\
0 & & 0 & 0_{1} \\
& \vdots & \ddots & \vdots \\
0 & & 0 & 0_{\operatorname{dim}\left(\mathrm{CUD}_{i}\right)}
\end{array}\right) .
$$

The number of lines of this weight matrix is given by the size of the original PCF $\left(\operatorname{dim}\left(\bar{\Lambda}_{i}\right)\right)$ to which we $\operatorname{add} \operatorname{dim}\left(\mathrm{CUD}_{i}\right)$ CSFs necessary for satisfying the CUD condition. In the deconstrained limit case, the weight matrix is a rectangular unit matrix of size $\left(\left[\operatorname{dim}\left(\bar{\Lambda}_{i}\right)+\operatorname{dim}\left(\operatorname{CUD}_{i}\right)\right] \times \operatorname{dim}\left(\bar{\Lambda}_{i}\right)\right)$

$$
\mathbf{D}^{i}=\left(\begin{array}{cccc}
1 & 0 & & 0 \\
0 & 1 & & 0 \\
& \vdots & \ddots & \vdots \\
0 & 0 & & 1 \\
0 & 0 & & 0_{1} \\
& \vdots & \ddots & \vdots \\
0 & 0 & & 0_{\operatorname{dim}\left(\mathrm{CUD}_{i}\right)}
\end{array}\right) .
$$

Adopting the weight matrix, the expression of a submatrix block $\left(\left(h_{i}+1\right) \times\left(h_{j}+1\right)\right)$ involving two different deconstrained PCFs can be obtained by a simple matrix multiplication

$$
\left\langle\left\{\Phi_{1}^{i}, \ldots, \Phi_{h_{i}}^{i}, \bar{\Lambda}_{i}^{d}\right\}|O|\left\{\Phi_{1}^{j}, \ldots, \Phi_{h_{j}}^{j}, \bar{\Lambda}_{j}^{d}\right\}\right\rangle=\left(\tilde{\mathbf{D}}^{i}\right)^{t} \tilde{\mathbf{O}}^{j} \tilde{\mathbf{D}}^{j}
$$

involving the counter-transformed weight matrix

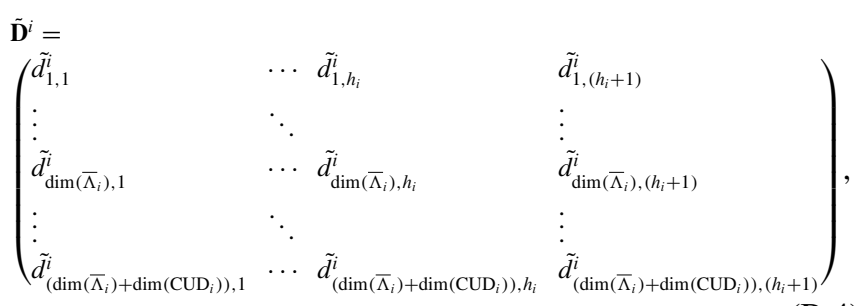

a similar one for $\tilde{\mathbf{D}}^{j}$, and the matrix representation of the selected operator $\tilde{\mathbf{O}}$ (21). The weight matrix formalism introduces the possibility of doing the simultaneous counter-transformation, associated to the biorthonormal transformation, of the expansion coefficients of each element of the deconstrained PCF. As each column of the original weight matrix (B.1) represents the expansion coefficients of each element, i.e. $\Phi_{1}^{i}, \ldots, \Phi_{h_{i}}^{i}$ and $\bar{\Lambda}_{i}^{d}$, of a deconstrained PCF in the original CSF space, each column of the countertransform weight matrix (B.4) gives the representation of these elements in the new CSF space expressed in the biorthonormal orbital basis. Another advantage of the weight matrix formalism is that we are able to treat, without any distinction, an arbitrary degree of deconstraint. The price to pay is a simple matrix dimension adaptation. By allowing high degrees of deconstraint, we quickly get large weight matrices and the use of their sparse representation is welcome.

For simplifying the counter-transformation process associated to the biorthonormal transformation, the lscud program originally developed for closing a CSF expansion under de-excitation [20] was adapted to provide the sparse structure of the counter-transformed weight matrix $\tilde{\mathbf{D}}^{i}$ before performing the biorthonormalization by itself. This is made possible by the use of the Warshall's algorithm [54] applied to the adjacency matrix mapping the single de-excitation operator for all $l$-values within the CSF basis. The obtained transitive closure matrix of the single de-excitation operator corresponds to the mask of the $\tilde{\mathbf{D}}^{i}$ matrix that determines the position of the non-zero elements after biorthonormalization.

\section{References}

[1] Löwdin P O 1955 Phys. Rev. 97 1509-20

[2] Mok D K W, Neumann R and Handy N C 1996 J. Phys. Chem. $1006225-30$

[3] Johnson W R 2007 Atomic Structure Theory: Lectures on Atomic Physics (Berlin: Springer)

[4] Lindgren I 2011 Relativistic Many-Body Theory: A New Field-Theoretical Approach (Springer Series on Atomic, Optical, and Plasma Physics vol 63) (Berlin: Springer)

[5] Dzuba V A and Flambaum V V 2007 Phys. Rev. A 75052504

[6] Johnson W R, Safronova U I, Derevianko A and Safronova M S 2008 Phys. Rev. A 77022510

[7] Safronova M S, Kozlov M G, Johnson W R and Jiang D 2009 Phys. Rev. A 80012516

[8] Knowles P, Schütz M and Werner H-J 2000 Ab initio methods for electron correlation in molecules Modern Methods and Algorithms of Quantum Chemistry, Proceedings (NIC Series vol 3) ed J Grotendorst (Jülich: John von Neumann Institute for Computing) pp 97-179

[9] Bartlett R J 2010 Mol. Phys. 108 2905-20

[10] Das Madhulita, Das Madhusmita, Chaudhuri R K and Chattopadhyay S 2012 Phys. Rev. A 85042506

[11] Hylleraas E A 1964 The Schrödinger Two-Electron Atomic Problem (Advances in Quantum Chemistry vol 1) (New York: Academic) pp 1-33

[12] Sims J S and Hagstrom S A 2009 Phys. Rev. A 80052507

[13] Komasa J, Cencek W and Rychlewski J 1995 Phys. Rev. A 52 4500-7

[14] King F W, Quicker D and Langer J 2011 J. Chem. Phys. 134124114

[15] Roos B O 1987 The complete active space self-consistent field method and its applications in electronic structure calculations Advances in Chemical Physics; Ab Initio Methods in Quantum Chemistry: II ed K P Lawley (Chichester: Wiley) chapter 69 pp 399-445

[16] Vilkas M J, Ishikawa Y and Träbert E 2008 At. Data Nucl. Data Tables 94 650-700

[17] Ishikawa Y, Santana J A and Träbert E 2010 J. Phys. B: At. Mol. Opt. Phys. 43074022

[18] Carette T, Drag C, Scharf O, Blondel C, Delsart C, Froese Fischer C and Godefroid M 2010 Phys. Rev. A 81042522

[19] Weijo V, Manninen P, Jorgensen P, Christiansen O and Olsen J 2007 J. Chem. Phys. 127074106

[20] Verdebout S, Jönsson P, Gaigalas G, Godefroid M and Froese Fischer C 2010 J. Phys. B : At. Mol. Opt. Phys. 43074017

[21] Malmqvist P-Å 1986 Int. J. Quantum Chem. $30479-94$

[22] Olsen J, Godefroid M, Jönsson P, Malmqvist P- $\AA$ and Froese Fischer C 1995 Phys. Rev. E 52 4499-508 
[23] Froese Fischer C, Brage T and Jönsson P 1997 Computational Atomic Structure: An MCHF Approach (Bristol: Institute of Physics Publishing)

[24] Godefroid M, Jönsson P and Froese Fischer C 1998 Phys. Scr. T 78 33-46

[25] Froese Fischer C 1977 The Hartree-Fock Method for Atoms. A Numerical Approach (New York: Wiley)

[26] Lindgren I and Salomonson S 1980 Phys. Scr. 21 335-42

[27] Gaigalas G and Rudzikas Z 1996 J. Phys. B : At. Mol. Opt. Phys. 29 3303-18

[28] Gaigalas G, Rudzikas Z and Froese Fischer C 1997 J. Phys. B: At. Mol. Opt. Phys. 30 3747-71

[29] Komasa J and Rychlewski J 2001 Chem. Phys. Lett. 342185

[30] Godefroid M, Froese Fischer C and Jönsson P 2001 J. Phys. B: At. Mol. Opt. Phys. 34 1079-104

[31] Engels B 1993 Theor. Chim. Acta 86 429-37

[32] Chung K T and Zhu X-W 1993 Phys. Rev. A 48 1944-54

[33] Fleming J, Godefroid M, Bell K L, Hibbert A, Vaeck N, Olsen J, Jönsson P and Froese Fischer C 1996 J. Phys. B. At. Mol. Opt. Phys. 29 4347-63

[34] Głowacki L and Migdałek J 2006 J. Phys. B: At. Mol. Opt. Phys. 391721

[35] Kramida A and Martin W C 1997 J. Phys. Chem. Ref. Data 26 1185-94

[36] Irving R E, Henderson M, Curtis L J, Martinson I and Bengtsson P 1999 Can. J. Phys. 77 1-7

[37] Schnabel R and Kock M 2000 Phys. Rev. A 61062506

[38] Martinson I, Gaupp A and Curtis L J 1974 J. Phys. B: At. Mol. Phys. 7 L463

[39] Träbert E and Curtis L J 2006 Phys. Scr. 74 C46-54
[40] Fuhr J R and Wiese W L 2010 J. Phys. Chem. Ref. Data 39013101

[41] Yan Z-C and Drake G W F 1995 Phys. Rev. A 52 R4316-9

[42] Yan Z-C, McKenzie D K and Drake G W F 1996 Phys. Rev. A 54 1322-7

[43] Lindgren I and Morrison J 1982 Atomic Many-Body Theory (Springer Series in Chemical Physics vol 13) (Berlin: Springer)

[44] Hill R N 1985 J. Chem. Phys. 31173

[45] Yan Z-C, Tambasco M and Drake G W F 1998 Phys. Rev. A 57 1652-61

[46] Sturesson L and Froese Fischer C 1993 Comput. Phys. Commun. $\mathbf{7 4} 432$

[47] Bransden B H and Joachain C J 1983 Physics of Atoms and Molecules (London: Longmans Green)

[48] Sakurai J J 1985 Modern Quantum Mechanics (Menlo Park, CA: Benjamin-Cummings)

[49] Bauche J 1969 Physica 44 291-300

[50] Judd B R 1963 Operator Techniques in Atomic Spectroscopy (New York: McGraw-Hill)

[51] Aufmuth P 1982 J. Phys. B: At. Mol. Phys. 15 3127-40

[52] Jönsson P, Gaigalas G, Bieroń J, Froese Fischer C and Grant I P 2013 Comput. Phys. Commun. at press

[53] Froese Fischer C, Tachiev G, Gaigalas G and Godefroid M R 2007 Comput. Phys. Commun. 176 559-79

[54] Koshy T 2003 Discrete Mathematics with Applications (New York: Academic)

[55] Malmqvist P-Å, Roos B O and Schimmelpfennig B 2002 Chem. Phys. Lett. 357230 